\title{
Understanding Tabletop Games Accessibility: Exploring Board and Card Gaming Experiences of People who are Blind and Low Vision
}

\author{
Adrian Bolesnikov \\ Carleton University, Ottawa, Ontario, \\ Canada \\ adrian.bolesnikov@carleton.ca
}

\author{
Jin Kang \\ Carleton University, Ottawa, Ontario, \\ Canada \\ jin.kang@carleton.ca
}

\author{
Audrey Girouard \\ Carleton University, Ottawa, Ontario, \\ Canada \\ audrey.girouard@carleton.ca
}

\begin{abstract}
Gaming accessibility research for blind or low vision (BLV) communities largely focuses on digital games. There is a need for designers to understand BLV's experience with tabletop games that involve the player's physical interaction. In this study, we investigate BLV individuals' experience with the accessibility of tabletop games. We conducted semi-structured interviews with 15 BLV participants and found four themes that uncovered participants' tabletop gaming experiences: (1) properties of inaccessible games, (2) outcomes of inaccessible games, (3) properties of accessible games, and (4) outcomes of accessible games. Our findings demonstrate a richness and variety in BLV individuals' tabletop gaming experiences. By providing discussions on the state of tabletop game interactivity and design recommendations, our work assists the creation of accessible tangible games that make use of digital information and physical forms by affording designers the opportunity to understand how inaccessible interactions in tabletop games affect BLV populations.
\end{abstract}

\section{CCS CONCEPTS}

- Human-centered computing $\rightarrow$ Accessibility; Accessibility; Empirical studies in accessibility; Accessibility design and evaluation methods.

\section{KEYWORDS}

Tabletop games, Blind, Low vision, Board games, Accessibility

\section{ACM Reference Format:}

Adrian Bolesnikov, Jin Kang, and Audrey Girouard. 2022. Understanding Tabletop Games Accessibility: Exploring Board and Card Gaming Experiences of People who are Blind and Low Vision. In Sixteenth International Conference on Tangible, Embedded, and Embodied Interaction (TEI '22), February 13-16, 2022, Daejeon, Republic of Korea. ACM, New York, NY, USA, 17 pages. https://doi.org/10.1145/3490149.3501327

\section{INTRODUCTION}

Board and card games are a popular activity for both sighted and people who are blind or low vision (BLV) [29, 92]. Through

Permission to make digital or hard copies of all or part of this work for personal or classroom use is granted without fee provided that copies are not made or distributed for profit or commercial advantage and that copies bear this notice and the full citation on the first page. Copyrights for components of this work owned by others than ACM must be honored. Abstracting with credit is permitted. To copy otherwise, or republish, to post on servers or to redistribute to lists, requires prior specific permission and/or a fee. Request permissions from permissions@acm.org.

TEI '22, February 13-16, 2022, Daejeon, Republic of Korea

(c) 2022 Association for Computing Machinery.

ACM ISBN 978-1-4503-9147-4/22/02 ..\$15.00

https://doi.org/10.1145/3490149.3501327 these games, players experience a heightened sense of comradery, teamwork, and autonomy [74]. Along with their positive social aspects, tabletop games are an effective educational tool with numerous applications ranging from teaching mathematics and drug awareness to developing social skills for neurodivergent children $[54,58,77,94]$.

However, modern game design-digital and tangible-has been largely uninformed by the experiences of BLV population. For instance, board and card games communicate information through colour, text, and images, which creates an accessibility barrier for those who are BLV [3, 74]. In addition, modern games are frequently incompatible with assistive technologies, and their designs include three-dimensional concepts that are difficult to express properly for those without vision [4]. The prevalence of inaccessible game designs is surprising given that many people with disabilities play games [96]. As a result of inaccessible games, BLV players report poor gaming experiences, including lowered quality of social interaction, stifled autonomy, and decreased game engagement $[3,28,65,78,95]$.

Research on accessible digital gaming is making advances for those with motor impairments or neurodivergent individuals [40, $49,52]$ and it is making similar strides for BLV players in digital environments [3,4]. Efforts surrounding accessible tabletop gaming, however, have proven to be less numerous. Much of the work has taken shape in translating tabletop games to digital $[28,78]$ and designing tabletop games for education (vs. entertainment) [77], let alone the scarcity of work investigating BLV individuals' tabletop gaming experiences [74].

Given this background, we sought out to bridge the knowledge gap within accessible gaming by investigating the experiences of BLV players with tabletop games. Our goal is to influence a shift towards a more ability-based design model for the tangible gaming industry. We posed the following research question:

Research Question (RQ): Regarding game accessibility, what are the tabletop gaming experiences of people who are BLV?

To address this question, we conducted semi-structured interviews with $15 \mathrm{BLV}$ individuals who shared their experiences and opinions on the current state of accessibility in tabletop gaming. Through thematic analysis, participants highlighted the themes including (1) properties of inaccessible gaming, (2) outcomes of inaccessible games, (3) properties of accessible games, and (4) outcomes of accessible games. Our study contributions are the following:

- We expand on existing guidelines that outline what make tabletop games (in)accessible. We augment existing guidelines by introducing subtleties within already identified 
(in)accessible properties and offer new (in)accessible properties of tabletop games.

- We identify specific outcomes from playing (in)accessible tabletop games as opposed to prior work that do not differentiate digital and tabletop game playing.

- We directly engage BLV players to learn about their experience and promote the culture of inclusive research.

Overall, our study is of high interest to Human-Computer Interaction (HCI) and TEI communities. Our work will inform the communities on how they can incorporate the needs and abilities of BLV players into all aspects of tabletop and subsequently tangible game design from game mechanics to game materials and rulebooks through a series of accessible design recommendations. Furthermore, our work will inform the design of accessible products by leveraging an inquiry into the feasibility of current trends in tangible gaming regarding the digitization of games.

\section{BACKGROUND AND RELATED WORK}

In HCI literature, the term tangible game often describes any game in which the players can physically interact with and manipulate core mechanics and game elements [26, 32, 45, 57, 65]. Tangible user interfaces (TUI), however, are commonly understood to be those in which digital information can be interacted through the physical environment [8]. In our paper, we capture the accessibility experiences of BLV individuals with physical gaming styles and will therefore be using the term tabletop game to describe all non-digital games (e.g., board games, card games, paper-and-pen games). We will use the term tangible game to describe gaming that makes use of digitalphysical hybridization. Investigating the interactivity of tabletop gaming remains crucial to understanding tangible gaming as, with any TUI domain, one must have a clear perception of both the physical and digital designs separately to be able to merge the two.

We first discuss gaming accessibility and tangible user interface for BLV individuals. Secondly, we examine the current state of digital and tabletop gaming accessibility for BLV individuals to exemplify the gaps that exist within accessible tabletop gaming research.

\subsection{Game Inaccessibility and Tangible User Interface for BLV Individuals}

Despite a survey conducted by Accessibility Foundation reporting $92 \%$ of the survey participants with disabilities playing an average of 10 hours per week [25], most games often exclude BLV players due to inaccessible design $[37,96]$. These restrictions arise because many games require a constant awareness of their status and communication through visual elements and hidden information. While non-mainstream accessible games exist (e.g., audio and text-based games), BLV players often find these games less entertaining [3] and complicated and expensive to play [37]. Heron et al. [41] found players with low-vision can only play tabletop games in very specific circumstances, such as players who still retain a small amount of their vision or when an assistive aid is present. An aid like sighted assistance in itself has been reported to draw BLV players away from the gaming experience and dramatically reduce the overall game enjoyment as people with various levels of sight experience the world differently [56, 91].
The outcomes of playing inaccessible games are dismal at both social and personal levels. Inaccessible games can prevent BLV players from reaping the social benefits of group play [10, 32, 37]. This is due to feeling marginalized and a burden to other players [37]. At a personal level, BLV players struggle to use games to relieve their stress and lift their moods [69] and they can experience a reduced personal sense of autonomy [74].

In the past, tangible interaction has supported the equal participation of groups commonly negatively impacted by inaccessible design (e.g., children, [5]; seniors, [55]). As such, tangible user interface (TUI) research for BLV users has received significant attention in recent years, notably when using TUIs to aid BLV users in their exploration of virtual spaces and graphical user interfaces (GUI) $[16,30,80]$. For instance, Tixier et al. [84] explored the use of a perceptual supplementation system that allows BLV users to experience a heightened sense of exploration in GUIs. Vetter et al. $[88,89]$ similarly explored the tangible adaptation of GUIs for BLV users through the context of perceiving digital music data presented in GUIs. TUI research for BLV has explored more unconventional means of interaction as well with interaction through deformation and wearable technologies [15, 20, 33, 71, 86]. Gollner et al. [36] highlighted the potential wearable TUIs have as a medium for assistive devices, in their case helping to bridge the technological communication gap deaf-blind people experience when using the Lorm alphabet. This amalgamation of literature alone demonstrates the capability tangible interaction has for supporting BLV lived experiences.

\subsection{Digital and Tabletop Game Accessibility}

2.2.1 Digital Game Accessibility. Digital game research has expanded greatly in recent years and presents a variety of methods for examining the accessibility of game design for BLV players. The creation of accessible design guidelines is a common practice in video game design due to its ability to be translated to a variety of technologies [9, 31, 44]. Andrade et al. [4] provided recommendations to address the unique needs of BLV players emphasizing the importance of spatial and object information and considering control both within the game and externally via assistive devices. Secondly, creating novel digital games is another popular research focus to address the needs of people with different disabilities $[6,60,63,70,72,79,82]$. Hernandez et al. [39, 40] reported the feasibility of custom exergame stations for action games promoting cardiovascular health for individuals with cerebral palsy [49]. Lastly, accessible digital gaming research has examined the potential of different adaptation techniques for existing games [38, 48, 95]. Attkinson et al.'s [7] examination of AudioQuake demonstrated how game designers can adapt accessibility of existing mainstream games at different levels such as online play, level editing, and auditory accessibility. [11]. Morelli et al. [61, 62, 64] have gone one step further by exploring the feasibility of tactile feedback for adapting digital exergames to support BLV players.

These works highlight substantial dedication to creative and unique solutions for digital gaming accessibility. While beneficial for digital experiences, this is unfortunate as the potential of these practices have yet to be given the same attention within tabletop game design. Topics introduced by the likes of Andrade et al. [4] 
regarding the representation of object information and Attkinson et al.'s [7] adaptations at different levels of gameplay are equally important for tangible games. This emphasizes the needed development of similar knowledge to create accessible tabletop games, the topic which we will now discuss.

\subsubsection{Tabletop Gaming Accessibility.}

Design Guidelines and Recommendations. While most accessible game design guidelines tend to focus on digital games, there exists several prominent studies for tabletop games $[58,68]$. In their guidelines, Tomé et al. [74] suggested that designers should strive for effective use of colour and contrast, tactile feedback, information design, game rules, and assistive technologies. Their approach is particularly noteworthy as it highlights the importance for its gameagnostic perspective on tabletop game design. Similarly, Heron et al. [42] presented a toolkit for evaluating the accessibility of tabletop games. They emphasized researchers can firmly assess accessibility of the large diversity of tabletop games by utilizing the Meeple Centred Design Heuristic Toolkit. The toolkit offered a breadth of considerations, ranging from visual (e.g., tactility, colour choice) and cognitive impairments (e.g., reading, game state complexity) to socioeconomic concerns (e.g., inclusive artwork, cost).

Novel Game Creation. Unlike digital gaming research, tabletop gaming research has mainly focused on the creation of accessible games for educational purposes [54,76]. Studies have found that different methods of tactile gameplay were effective in teaching BLV youth a variety of programming concepts $[1,47,50]$. Others have found that tabletop games can aid parents in teaching their children Braille [34]. Regal et al.'s [73] work presented a different approach of novel accessible game creation using a game-agnostic approach; they discussed the potential for tabletop game creation toolkits to aid children with visual impairments create their own movement-based games.

Existing Game Adaptation. Under this research focus, prior work has investigated numerous applications of game adaptation. Bhaduri et al.'s [12] work into the use of 3D printers for fabrication gameplay highlights the importance of the tangible nature, as participants suggested the use of fabrication tools for fixing or upgrading inaccessible games. Johnson et al. [46] explored a higherlevel means of tangible adaptation by presenting Game Changer, a game-agnostic adaptation toolkit that allows BLV players to set up a system that downloads unique adaptations to a computer system for each individual game they play. The system addressed the issues of unfair play and encouraged independent play, but it required sighted assistance and non-customizable level of feedback. In a like manner, Thevin et al. [83] explored the creation an augmented reality based adaptation toolkit to improve on the accessibility of tabletop games for BLV players. Thevin et al. expanded on these existing works, however, by not only including visual feedback to support participating of sighted players with BLV players but the ability for players to make digital editions.

Johnson et al.'s [46] work demonstrates another common trend in game accessibility research: digitization of tabletop games. Some have investigated the effect of digitization on factors like engagement $[81,90]$; others have examined the potential of complete translations of tabletop games into digital ones [26, 28, 65, 78]. Braley et al. [17] exemplified this possibility by discussing the process of translating an educational board game into a mobile version. While advantageous in providing accessible gaming experiences, the complete digitization of tabletop games ultimately removes the benefits tabletop gaming experience of playing with others. This highlights the importance of our work as it lends to the opportunity of creating accessible tangible games that maintain their physical nature.

In our study, we sought out to expand on the research area of design guidelines because establishing accessible design guidelines can significantly influence the latter two research areas. Prior efforts at designing existing guidelines and our own study share the same broad goal of identifying general accessibility challenges faced by BLV players with tabletop games. This broad approach is understandable given the nascent research area. We explored additional (in)accessible properties of tabletop games that can augment existing guidelines $[42,74]$. Can we introduce subtleties within each identified property or even new properties to make the design guideline as a whole more comprehensive and reliable?

Heron et al. [42] proposed heuristics to evaluate existing tabletop games on accessibility; yet their guidelines are derived from a relatively abled perspective. Tome et al. [74] established their guidelines from conducting a user testing with two tabletop game genres, which have already been modified to enable autonomous play in BLV players. Our study attempted to address these two weaknesses by directly involving BLV players who shared their natural game experience beyond two tabletop game genres. There exist trends in accessibility research to not directly involve BLV participants [22, 46, 54]. Our study contributes towards reinforcing the value of conducting inclusive research.

A more solid foundation of accessible tabletop gaming research requires a greater understanding of the general experiences of end users with different disabilities. It is vital for designers to have a proper understanding of the current level of interactivity in tabletop games from the end-user perspective before they can leverage the abundance of knowledge already gathered in fields such as tangible devices and digital gaming accessibility. We also sought out to identify specific outcomes from playing (in)accessible tabletop games. Prior work has not differentiated digital and tabletop game playing outcomes [37].

Against this background, we pose the following research question: Regarding accessibility, what are the tabletop gaming experiences of people who are BLV? By addressing our research question, we will be able to fill this gap and gain a deeper understanding of BLV individuals' attitudes and practices with tabletop games.

\section{METHODOLOGY}

\subsection{Participants}

We recruited fifteen participants (11 men and 4 women; 13 from North America and 2 from Europe) via social media platforms (Reddit at r/Blind, Facebook, Twitter), networks within the visually impaired community or snowball sampling technique. All participants were over the age of 18, comfortable with the English language, and with vision that could not be corrected through standard means such as glasses or contacts. Table 1 displays a summary of the study's participants' demographics. We did not require gaming 
Table 1: - Study Participant Demographics

\begin{tabular}{llll}
\hline Participant & Age & Level of Vision (Onset) & Assistive Devices \\
\hline P1 & 20 & Blind (at birth) & Braille, Screen Reader \\
P2 & 26 & Legally blind (at birth) & iPhone Voice-Over, White Cane, Screen Reader \\
P3 & 35 & Blind (at age 29) & Screen Reader, White Cane \\
P4 & 23 & Legally blind (at birth) & Screen Reader, Screen Magnifier \\
P5 & 30 & Blind, light perception (at age 25) & Screen Reader \\
P6 & 39 & Blind (at birth) & Text-to-Speech Software \\
P7 & 32 & Legally blind (at age 30) & None \\
P8 & 53 & Blind, light perception (at age 34) & White Cane, Screen Reader \\
P9 & 18 & Blind, light perception (at birth) & Screen Reader \\
P10 & 22 & Blind (at birth) & Screen Reader, White Cane \\
P11 & 54 & Blind (at birth) & Screen Reader, White Cane, Guide Dog, Braille \\
P12 & 31 & Low vision (at birth) & Screen Magnifier, Physical Magnifier \\
P13 & 25 & Blind (at birth) & Screen Reader, Braille Display, Guide Dog \\
P14 & 31 & Blind, minor light and colour perception in one eye (at & Screen Reader, Braille Display \\
& & age 10) & \\
P15 & 26 & Minor light/colour perception & Guide dog, Voice-Over \\
& & (at age 21) & \\
\hline
\end{tabular}

familiarity so that we could understand any barriers to accessibility for tabletop games. However, all participants indicated some gaming experience, with qualifiers such as daily or hobby play ("Avid”), occasional play ("Casual”) or little to no play because of accessibility limitations or lack of interest ("No"). Table 2 highlights a summary of participant's information regarding gaming history such as games played and genres covered. It is important to note that the games played only reflect games explicitly mentioned by name during the interview, several participants indicated having played many more tabletop games such as P8 who had previously "donated hundreds of dollars worth of games." Collectively, participants' gaming experience covered the eight genres of tabletop games (e.g., board, card, tile-based) [97]. We compensated participants for their time with a CAD 25 electronic gift card to the online retailer of their choice. Our local Research Ethics Board approved this study.

\subsection{Data Collection}

We collected data via semi-structured interviews with each of the participants. Twelve participants used Zoom, one participant used Google Meet, and two participants opted for phone calls. We recorded all interviews, and they lasted on average 39 minutes. The interviews questions centered on the topics of demographics, general gaming, tabletop gaming, and digital gaming. General gaming questions explored the participant's gaming experiences, while the two following sections dove in-depth into each gaming type. Topics of discussion included the importance of different senses when playing games, techniques for learning the foundations of new games, personal game adaptations for accessibility, and the impact of different assistive techniques on game experiences. We also asked similar questions on digital gaming to investigate the existence of any elements of accessible digital gaming that could be carried over to tangible games.

\subsection{Data Analysis}

We transcribed 5 interview recordings manually and 10 using the transcription software Trint [85]. We used Trint for interviews in which participants consented to us using the software to accelerate the transcription process. The lead researcher reviewed all automatic transcriptions for accuracy with the data prior to analysis.

The first and the second authors conducted reflexive thematic analysis on interview transcripts [19]. We followed the established guidelines by Braun and Clark [18]. First, each author engaged in data familiarization independently, reading and re-reading through interview transcripts. Second, two authors reviewed and coded two of the transcripts together to establish an initial code frame $[19,35]$. Then, the first and the second authors coded a second set of two interview transcripts independently using the established code frame and reconvened to discuss for discrepancies. Third, the researchers repeated the same process for the rest of the transcripts; at each stage, they reviewed and redefined codes and then grouped them into broader themes. Fourth, we refined these initial themes in terms of the degree to which they were the central organizing concepts that captured our participants' gaming experience.

Lastly, we theorized about the data by developing a thematic relationship between each theme. This was done based on the discussion of how each theme and subcategories can be related to each other and we generated our own theoretical codes to assign meaning to each relationship (i.e., "consist of" and "lead to") [87].

\section{FINDINGS}

In response to the RQ, we found four themes: properties of inaccessible games, outcomes of inaccessible gaming, properties of accessible games, and outcomes of accessible games. We break down each theme into subcategories such as rulebooks, game content, and inclusive design. Figure 1 and Figure 2 demonstrate the thematic relationship between the properties of (in)accessible games and the consequent outcomes. Some subcategories share similar labels 
Table 2: - Study Participants' Tabletop Gaming History

\begin{tabular}{|c|c|c|c|c|}
\hline Participant & Gamer & Tabletop Games Played & Genres Played & Play Group(s) \\
\hline P1 & Avid & $\begin{array}{l}\text { UNO, Chess, Checkers, Battleship, Monopoly, } \\
\text { Candy Land }\end{array}$ & $\begin{array}{l}\text { Board, Strategy, Card, } \\
\text { Pencil and Paper }\end{array}$ & $\begin{array}{l}\text { Family, Friends, BLV } \\
\text { Community }\end{array}$ \\
\hline $\mathrm{P} 2$ & Avid & $\begin{array}{l}\text { Rummy, UNO, Chess, Monopoly, Dungeons \& } \\
\text { Dragons, Magic the Gathering }\end{array}$ & Board, Card, Role-Playing & Family, Neighbours \\
\hline P3 & Avid & $\begin{array}{l}\text { Cards Against Humanity, UNO, Exploding } \\
\text { Kittens, Dominion }\end{array}$ & Card, Strategy & Family, Partner, Friends \\
\hline $\mathrm{P} 4$ & Avid & Chess & Board & Family \\
\hline P5 & Avid & Secret Hitler, Pandemic, Love Letter & Card, Strategy & Friends \\
\hline P6 & Avid & $\begin{array}{l}\text { Descent: Journeys in the Dark, Star Wards: } \\
\text { Imperial Assault, Root }\end{array}$ & $\begin{array}{l}\text { Board, Strategy, Dice, } \\
\text { Adventure }\end{array}$ & Friends \\
\hline P7 & Avid & $\begin{array}{l}\text { Cribbage, Pinochle, Monopoly, Scrabble, Code } \\
\text { Names, Clank! }\end{array}$ & $\begin{array}{l}\text { Board, Card, Adventure, } \\
\text { Tile }\end{array}$ & Partner \\
\hline P8 & Avid & $\begin{array}{l}\text { UNO, Hearts and Spades, Roll Player, The } \\
\text { Quacks of Quedlinburg, Twilight Imperium, } \\
\text { Food Chain Magnate }\end{array}$ & $\begin{array}{l}\text { Board, Card, Strategy, } \\
\text { Dice }\end{array}$ & $\begin{array}{l}\text { Family, Friends, BLV } \\
\text { Community, Gaming } \\
\text { Community }\end{array}$ \\
\hline P9 & Casual & $\begin{array}{l}\text { Dungeons \& Dragons, Dominoes, Battleship, } \\
\text { UNO }\end{array}$ & $\begin{array}{l}\text { Board, Card, Role-Playing, } \\
\text { Tile }\end{array}$ & Friends \\
\hline $\mathrm{P} 10$ & Casual & Monopoly, Dominoes, Battleship, UNO & $\begin{array}{l}\text { Board, Pencil and Paper, } \\
\text { Tile }\end{array}$ & Family, Friends \\
\hline P11 & No & Scrabble, Cribbage, Monopoly, Timeline & Board, Card, Tile & Family, Friends \\
\hline $\mathrm{P} 12$ & Casual & $\begin{array}{l}\text { Battleship, Chess, The Game of Life, Sorry!, } \\
\text { Monopoly, Dungeons \& Dragons }\end{array}$ & $\begin{array}{l}\text { Board, Role-Playing, } \\
\text { Pencil and Paper }\end{array}$ & Family, Partner \\
\hline P13 & Casual & Cards Against Humanity, Scrabble & Card, Tile & Friends \\
\hline P14 & No & Othello & Board & Friends \\
\hline P15 & No & Yahtzee, Cranium & Card, Dice & Friends \\
\hline
\end{tabular}

in name only and we indicate this through matched color; each captures experiences that are unique to (in)accessible gameplay and outcome. For participants quotes, we removed filler words to enhance readability.

\subsection{Properties of Inaccessible Games}

Participants identified a complex relationship of different factors that, at different severities and frequencies, lend to a tabletop game being (in)accessible. Our participants reported how the design of a tabletop game's rulebook and content as well as their experiences with industry and community support towards accessibility have created inaccessible games.

4.1.1 Inaccessible Rulebooks. Inaccessible gaming rulebooks commonly act as barriers to access before games have even started. For example, P10 indicated how they often opt for sighted assistance when learning physical game rules as the typed instructions that come with the games are rarely available in Braille.

Five participants indicated that reading online manuals was their method of choice when learning the rules of a new board game rules, but the flawed creation of those manuals had several accessibility issues. P8 commented that game developers often create rulebooks with numerous images or as one single image that makes it difficult to use Optical Character Recognition (OCR) and make using OCR "a real hassle." P5 echoed this sentiment: "For instance, a lot of publishers still put their rulebooks as PDF images. And those are not accessible. The text is basically not accessible. They either scan the rulebooks, or they just use some sort of export to visual printer type of thing, which basically converts the rulebooks into images."

When discussing tabletop games that required the construction of objects, P10 indicated that "there's nothing you can do because you're to see the image in order for you to build a structure." P5 continued this idea as they indicated that when there are icons and images necessary to convey game information then "the screen reader ignores that icon."

4.1.2 Inaccessible Game Content. Participants defined barriers to the tabletop games in terms of text, colour, sound, touch, and game mechanics.

Text. P10, P11 and P12 indicated that the use of text in tabletop games increases the level of difficulty for BLV players. As a result of the text being too small, BLV players make the physical effort to get closer to the text or pausing the game entirely to read it. P7 shared that the added physical effort made them abandon games: "I play [board games] less now, mostly because I have to get really close to the board. That's annoying, right?" P12 also discussed the barrier introduced by ornamental fonts: "Well, going back to that [Dungeons and Dragons] game, like I said, the text was kind of difficult to read, but it was a font. I know that kind of went with the theme of the game, but I think if the text were easier to read and bolder and they could just kind of decorate around the text to keep it within that theme. . .that would help." 


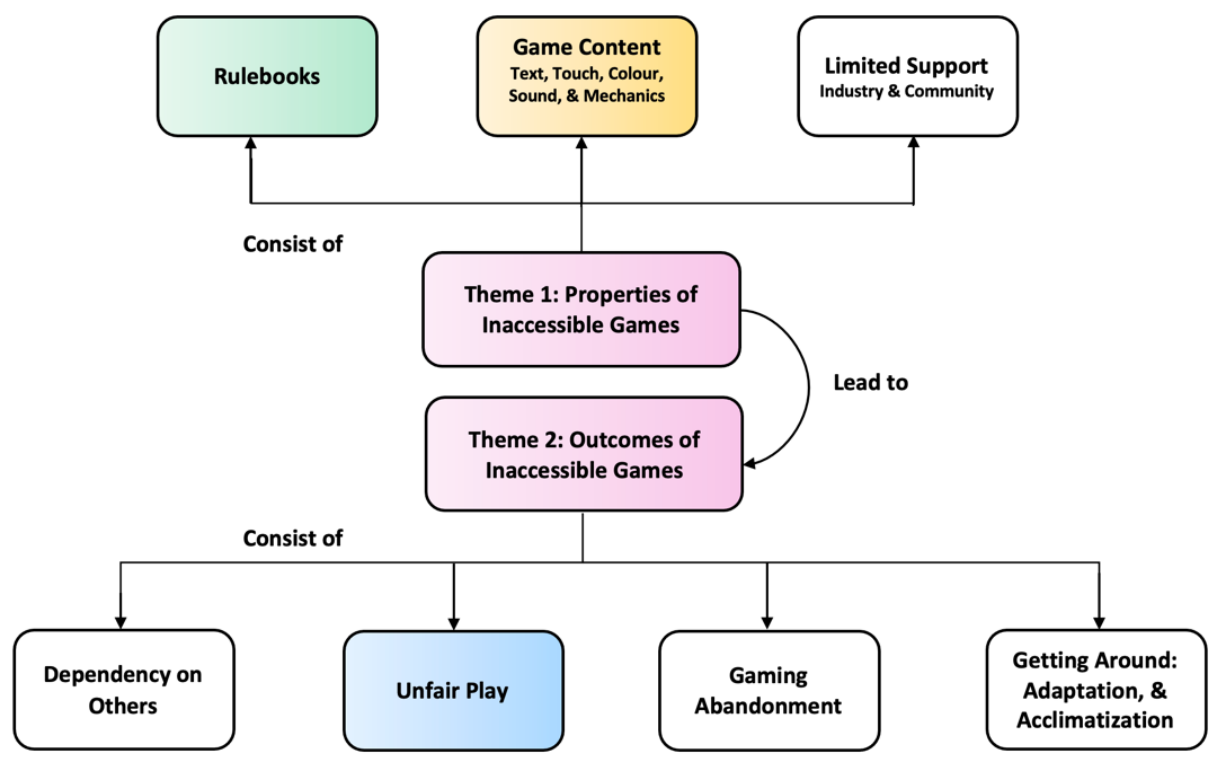

Figure 1: Thematic Relationship between Themes for Inaccessible Games

Colour. P5 and P6 commented on tabletop gaming's reliance on colour to communicate information to players. In particular, participants with colour perception are often faced with difficulty in differentiating game elements with poor colour contrast. Both P2 and P12 alike commented that low contrasting colour often leads to the blending of visual elements. P7 explained, "if you're having a bright colour on another bright colour, I might not be able to see it whatsoever. And so I would hate it, I do need to have high contrast." P5 and P6 comparably discussed the disadvantage colour blind and sighted players can face when there is a lack of modalities beyond colour and suggested using a combination of elements like colour and shape to differentiate game pieces.

Touch. Despite being typically a useful substitute for vision, several participants have encountered issues with the use of touch in tabletop gaming. P11 said, "most games are not tactilely able. You're not able to feel where to go on the board. You're not able to feel where other people are on there, or to even like, to know what to do when you get to certain places. . that's the most important thing is that you can manipulate things like anyone else does."

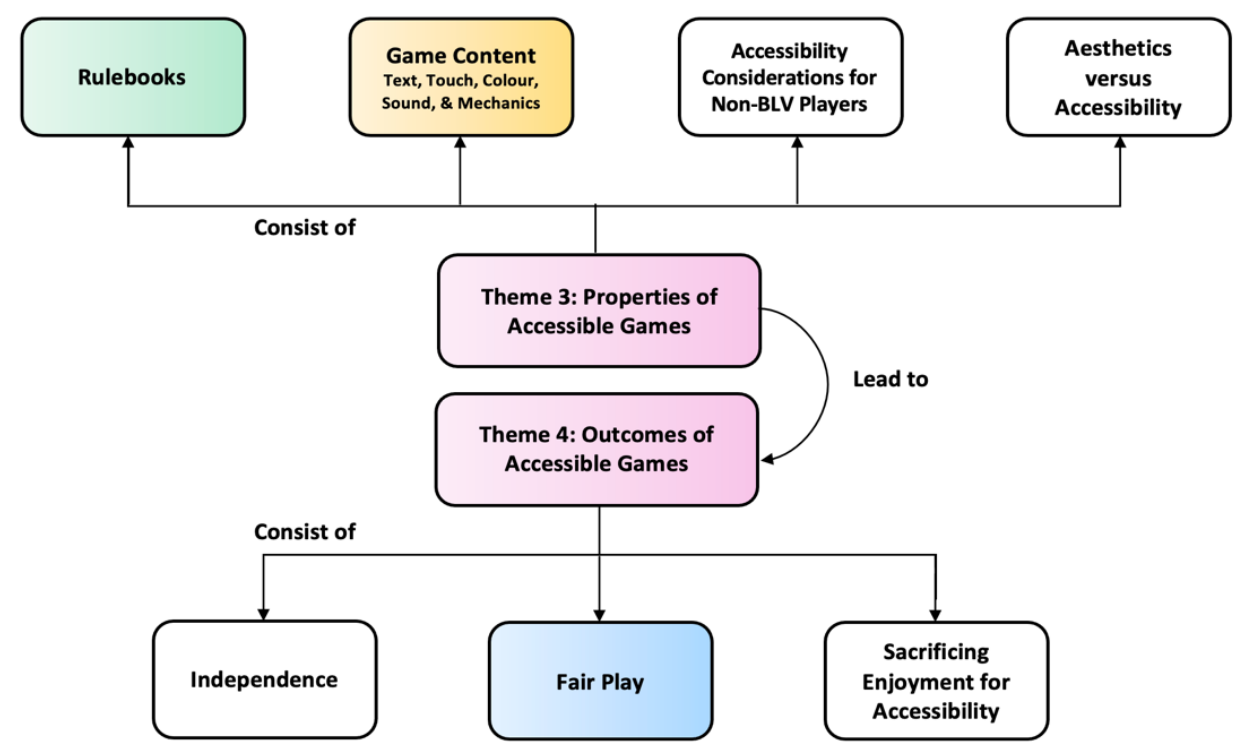

Figure 2: Thematic Relationship between Themes for Accessible Games 
While tabletop games make an abundant use of tactile game elements, participants highlighted that most tabletop games do not use physically distinct pieces. This prevents BLV players from being able to make use of the sense of touch to distinguish parts of the game and prevents game progression. Participants also noted a lack of tactile methods of communication such as Braille for text-based card games. This negatively influenced some participants' game progress because they unwillingly moved game components when feeling the board for tactile information.

Sound. Two participants discussed barriers caused by flawed sound use in digital games. P1 indicated that the absence of sound cues in games leads to the reliance on sighted assistance; P3 highlighted the absence of audio descriptions as the main barrier: "the game designers relied heavily on just the dialog and character feedback in order to kind of tell the story as opposed to what everyone else gets visually." The presence of sound alone may not be always enough to make a game accessible, but rather the use of it to provide insight into the entire environment. While highlighted as a barrier for digital games, four participants shared that they would have appreciated more uses of what they refer to as oriental sound, or sound used to help guide users in a physical space, in tabletop games akin to digital games. Thus, these same digital inaccessible properties are likely to exist within tabletop games that do make use of audio whether for multimodality or as a core game mechanic.

Game Mechanics. All participants indicated accessibility concerns surrounding game mechanics. Five participants noted the difficulty fast-paced time actions pose for BLV players, regardless of sighted assistance. P8 discussed time-based games as being the greatest deterrent from playing certain games due to their level of sight: "as far as inhibition because of visual limitations, I'm very highly against it. And I will just simply not play a game that has [timed actions] as a part of it." Five participants expressed that, while not as serious a deterrent, timed actions add an extra amount of pressure. P3 said this pressure often is a result of requiring extra time to perceive the information before them. "Trivia Crack [a digital game] where you have 30 seconds to answer a trivia question, but then you have to take into account how long does it take a user to listen to the question through a screen reader and then listen to the individual options?"

The presentation of information also presented accessibility concerns. For seven participants, tabletop games with excessive amounts of information, such as Settlers of Catan, introduce issues of cognitive load. Three participants expressed cognitive load results from games that make use of a purely visual coding to communicate board game state information. P15 discussed this issue as having an impact on their ability to make decisions in-game: "whatever choices I choose to make during a round based on my current context. I can't look at the board and think, 'well this person is ahead so I should take this person out or something." For others, cognitive load results from games requiring hidden and personal inventory management. Games, such as Bonanza, require players to keep track of their personal private information and remain aware of other players' inventories. P8 expanded on this by stating that if games rely on only one or two modalities, hidden information adds an additional layer of difficulty on top of existing accessibility concerns.
4.1.3 Limited Support. Eleven participants expressed the state of gaming industry and community support as potential causes for barriers to games. P10 explains the availability of many "accessible" games as being coincidental: ". . I think [Dominoes] was something that came out accessible, but I don't think they were thinking, oh, let's make this accessible." P10 cited dominoes as being one of the most accessible games by default for BLV players as players can identify all game components through distinct grooves [67]. Some participants were dissatisfied with the lack of accessible games, with P9 expressing: "as default [game developers] should attempt to make everything accessible as possible."

Relatedly, there was a finance aspect that made a limited number of accessible games inaccessible: "Monopoly is really fun but there is an official Braille board that is like eighty dollars, it's crazy" (P1). Participants who did inform us about available accessible games also often did so with the caveat of there being few genuinely good solutions in their opinion. P1 provided an example of the lack of appropriate adaptations by indicating that a common solution is to simply add Braille without much consideration: "The first thing that a person may say is, oh, let's just put Braille in it. You know, um, the thing about Braille is yes it's very nice, but it could be really inconvenient."

In addition to industry support, two participants also mentioned limited support from the sighted gaming community for BLV players: "I would say the most barriers to face are when playing with sighted people since they might not really think of what's a blind person may need. So, they would, for example, just put the card in the centre of the table and wouldn't really have in mind the blind person, that they would need to put up a hand to see what card it is" (P9).

While the current support was less than stellar, there was the rise of the gaming industry's and community's awareness of inaccessibility. P2 and P10 discussed how major retailers such as Amazon and Target have slowly begun to provide popular accessible games such as Braille UNO. P3 likewise informed us that there is an increase on the part of BLV associations and groups to provide gaming support. P3 provided the example: "LightHouse for the blind here in San Francisco. They have an annotation store which sells accessible media and things, and part of that is like pre-Braille playing cards and free Braille."

\subsection{Outcomes of Inaccessible Games}

Our participants similarly discussed the impact that inaccessible design of a tabletop game has on their experience with it. We found four outcomes of inaccessible games experienced by our BLV participants: the dependency on others, gaming abandonment, unfair game experiences, and techniques to getting around gaming barriers.

4.2.1 Dependency on Others. P5 and P8 indicated that the patience of others was vital to tabletop gaming. This was a result of elongating the play sessions so that they may have the time to orient themselves. P15 had to force her counterparts to slow down because she is "the one that's taking the most time." Four participants indicated that they require sighted assistance to play parts of the game for them. P1 explained, while playing Monopoly, their assistant will make the decisions for them: "So sometimes guiding me on 
what cards to choose and what to do next is not really me choosing myself, but sometimes people will choose this one." P12 expanded on the reduced enjoyment as a result: "So in a sense it's kind of like they're playing the game for me and it kind of makes it less fun."

Eight participants relied on sighted assistance for overcoming issues such as inaccessible text to provide a "kind of visual context" (P15). P6, however, expressed a need to be selective in choosing games to avoid sighted assistance whenever possible to protect the integrity of the game experience: "I need to ask a lot of questions, many of which may provide the other players with information about my intent either now, or turns later, if I'm executing some sort of plan that I am formulating based on what I know and the questions I've asked from that turn in prior turns.'

4.2.2 Gaming Abandonment. Six participants felt a need to completely give up on their gaming experiences because of their level of vision. P3 and P8 played less and began to avoid games upon the deterioration of their level of sight. P14 shared that it has been so long since they quit physical gaming due to their level of vision that they can no longer imagine physical games being enjoyable. P7 explained: "I game a lot less and I'm less willing to play new games. I'm just too afraid to have other people know how bad my sight is." P13 demonstrated that this attitude has also impacted their willingness to adapt games. "No, but I think this is mainly because we just kind of avoid playing games where that kind of thing would happen."

Two participants indicated, however, that they may pursue other means of experiencing the elements of certain games. P3 said: "I seek out YouTube channels that recap a lot of the games I never got to play." P4 had a similar suggestion: "I suppose I could just Google it if I really want it to know." Accessibility barriers to tabletop games for BLV players can lead to players giving up on experiencing them first-hand if they are not willing to tolerate impacted games.

4.2.3 Unfair Play. Four participants expressed concerns regarding the unfair play that comes as a result of inaccessible gaming. Looking at a higher level, $\mathrm{P} 4$ shared experiences of being at a disadvantage due to limited accessibility: "I would say that for me at least, I have to put in a little extra effort than the average player."

As explained by $\mathrm{P} 8$, this disadvantage is a detraction from the competitive nature of certain games. They said: "if you share [cards] with other people, then everybody knows what you have." P5, however, explained unfair play as a result of the lack of modalities to communicate information. They provided the example of those who are colour blind: "Maybe some sort of colour blindness, it can certainly put you at a disadvantage as a visually impaired player." P15, however, suggested that the disadvantage can actually be for the sighted players who do not receive the extra assistance: "so they kind of give me an analysis of the board, whereas others wouldn't get that."

\subsubsection{Getting Around.}

Adaptation. Twelve participants made innovative adaptations to overcome barriers and they indicated that it is challenging to adapt a game to be completely accessible. P5 described the issues using an Alexa adaptation for a board game: "Alexa would just say, 'now grab the cards.' And since I can't see that I still would require someone sighted to be able to grab those cards out of the box. So I'm not being fully independent." P14 extended this point by informing us that often blind-friendly audio games will have a built-in text-tospeech (TTS) that would conflict with their own screen reader. P3 continued this idea by suggesting that they must re-adapt certain adaptations to address new issues: "One of the things that I found with Exploding Kittens was after playing it enough with my friends, because I Braille the cards, the Braille is popped out through one side, but it's punched in through the back. And they can look at the back and they can see what the upcoming card is based on the Braille."

The process of adaptation had a variety of its own barriers, with the time spent on adaptation $(n=5)$ being the greatest challenge. P2 said: "I haven't really had the motivation out there to go and spend three, four, five hours trying to grab something that may work then draft up a bunch of prototypes before setting on something." Other barriers included the limited transportability of the adapted games due to the increased weight and number of game pieces, as well as expenses to purchase materials for adaptation.

Three participants reflected on how game adaptation was a collective effort that involved their family and friends. P11 said: "I would do the Brailling, but [my family] would mark out the board. They would make sure, you know, the cards were labelled so I could play it too." Similarly, P13 shared their experience adapting Cards Against Humanity with their friends: "before they came out with an accessible version of cards against humanity me and a couple of my friends work together and Brailled the entire game. It was very arduous. It took a while." They did clarify, however, that despite the time-consuming nature of the adaptation, they found the process enjoyable: "we made a game up, and it was kind of entertaining. We all wanted to play the game, so it was worth it."

Acclimatization. Five participants indicated that, when faced with accessibility barriers, they opt to acclimate to the inaccessible gaming environment as best as possible. P14 shared they would simply try to use their better eye to make out the elements on a game board. P1 mirrored P14 in their own gaming experiences. "Candy Land, for example, is really big you just move your piece across the board on the colours and the colours are relatively large. So, if I look close enough, I can see, okay, that's the next blue tile."

Unlike P1 and P14, P2 discussed how they acclimatized to tabletop games in two ways: they played the parts of the game that were accessible enough and memorized the input needed to circumvent the troubling gameplay. P7 echoed the use of memorization to acclimate to inaccessible games but warned that it is not the ideal solution either: "That's another issue that like Magic the Gathering has is, I had that issue before I had the vision problem, because of how complex the characters are. But I don't have it memorized. So, games that there's little to memorize, you just play the game. Those are easy for me." Acclimatization, while unfortunate, demonstrates yet another level of creativity BLV players have innately when presented with inaccessibility barriers. Table 3 lists accessible and inaccessible games out-of-box and adaptations made by participants to make certain games accessible.

\subsection{Properties of Accessible Games}

We now report on the elements that would make both rulebooks and games accessible, considerations for inclusive design, and the 
Table 3: - (In)Accessible Games and Adaptations Made by Participants

\begin{tabular}{lll}
\hline Accessible Games Out-of-Box & Type & Reason \\
\hline Dominoes & Tabletop & Its game elements are physically distinct. \\
Monopoly & Tabletop & It has an official Braille board. \\
UNO & Tabletop & It has an official Braille version. \\
Battleship & Tabletop & Its game elements are physically distinct. \\
Scrabble Travel Edition & Tabletop & Its game elements are physically distinct. \\
Last of Us Part II & Digital & It allows players to pause game to allow for planning tactics. \\
\hline \hline Inaccessible Games Out-of-Box & Type & Reason \\
\hline Trivia Crack & Digital & It gives players 30 seconds to answer a trivia question. It \\
& & does not consider BLV players who needs to use a screen \\
& reader to listen to the question and the individual options \\
Settlers of Catan & Tabletop & It presents players with excessive amounts of information \\
Bonanza & Tabletop & It requires players to keep track of their personal private \\
& & information and remain aware of other players' inventories. \\
\hline \hline Adaptations Made & Type & Reason \\
\hline [Game name not provided] & Tabletop & BLV players adapted Alexa to announce to them the game \\
Exploding Kittens & Tabletop & BLV players brailed cards. \\
Cards Against Humanity & Tabletop & BLV players brailed cards. \\
\hline
\end{tabular}

balance between aesthetics and accessibility. We introduce a rich insight into participant opinions of these topics and their ideation for potential solutions to barriers addressed earlier.

4.3.1 Accessible Rulebooks. In a like manner to discussing the limitations, participants discussed the importance of creating simple and clear instructions. In P11's opinion, "I think also when you write the game, when you write the description of the game and the instructions of it, that it's clear and simple language and that it's in accessible form." P2 and P11 added that Braille rulebooks was also an ideal option: "Braille UNO has four UNO sized cards that came with it, each one has one sentence about how to play the game. There are four sentences. You're set, you can play all you want. It teaches you everything in four sentences" (P2).

Nine participants expressed the interest in a standardized, accessible medium for receiving game instructions. They highlighted the use of accessible websites for accessing rulebooks, particularly those that make proper use of HTML: "There are some publishers that also put the rulebooks in HTML form on their website. That's the most accessible way of getting through things" (P5). Participants also mentioned the prospect of using mobile devices and emerging techniques such as Quick Response $(\mathrm{QR})$ codes to present instructions. P8 shared, "if there was something someone had an accessibility part of their game and their website or had a scannable QR code card, and it would just send your iPhone or whatever you use to a website to be able to read the rules." This idea was appealing due to the customizability with accessibility features present in mobile phones: "maybe making an app or different categorize board game rules... alphabetize index, a board game, manual or you can look it up on your own device. That's comfortable for you" (P12).

\subsubsection{Accessible Game Content.}

Text. Participants had a variety of ideas for making the use of text more accessible. Common ideas included the use of large print or simplistic fonts. Four participants emphasized the potential Braille has when improving the accessibility of text in tabletop games by adding an extra information modality.

Four participants emphasized that textual elements, be it physical or digital, should be compatible with assistive technologies. Four participants highlighted technologies like Screen Readers, Seeing $\mathrm{AI}$, and OCR to play games. P3 explained their reliance on these technologies particularly for card games, "I'll use seeing AI or the OCR on the cards to read text to me if I have to read a card really quick or as quickly as I can. And that's basically all I've been using."

Colour. We report on the importance of conscious colour choice to improve accessibility for those who are BLV. Five participants expressed their concern over the lack of contrast. P12 expressed low colour contrast difficulties: "The little symbols on the cards, these cards, have coin symbols and other ones here with the same colour. So, I kind of got confused on what symbol was what because at first glance, you know, they're the same colour." Similarly, P5 shared the benefit high contrast has in making different elements distinct, "even with, in terms of accessibility, people with limited vision can benefit from colours, different distinguishing variations of it."

Touch. Nine participants described touch as the most common and effective facilitator for BLV players, particularly as means to "activate the internal visual channel." (P14). Texture was a particularly useful tool to communicate information to BLV players $(n=9)$. Adaptations like magnetized or Velcro tokens allowed them to manipulate the board without ruining the game state. P2 shared an 
example of how texture was useful for communicating spatial information when they adapted a chessboard by surrounding each square with paracord. P1 provided a similar adaptation suggestion with the use of guides such as a tactile key to help BLV players manage different textures used in a tabletop game. P14 explained the importance of texture to BLV players: "texture is to the blind as colour is to the sighted."

Finally, four participants noted the importance of having physically distinct game elements. Tabletop games such as Dominoes, Battleship, and Scrabble Travel Edition, are seen as accessible outof-the-box because many of their game elements are physically distinct, which BLV players found to be vital.

Sound. Similar to tangibility, nine participants indicated that the proper use of audio can act as an excellent substitute to elements that require visual perception. Concisely put, when discussing the potential of audio adaptations, P7 said, "This sounds very important because I can't see, but I can hear. So, if I can rely more on the sound then I'm set." P5 provided a more precise example when discussing the value of sound in adaptations for digital game accessibility: "Turn on the ability for the game to basically read the menu items for you, like new game options, et cetera."

Sound can play a major role in aiding game state communication and orientation for BLV players. Five participants stressed the benefit sound cues present for navigating within a gaming space. P10 proposed an idea for incorporating sound in tabletop games like Jenga that require dedicated movement around certain objects: "So like an indicator of how close you are or something, which pieces you can take maybe to make, you know. Some insight into the whole picture, that will be something very helpful."

Game Mechanics. Three participants discussed potential techniques for incorporating timed actions in an accessible manner. For example, P1 insisted that any time-based action should not be too involved, "all time-based actions, you have to make sure that the specific things you want to be done is relatively simple." Other participants suggested timed elements remain relatively slow paced and make use of turn-based mechanisms to ease the pace. For example, P3 drew upon the example of the Last of Us Part II in which accessibility features allow players to pause games to allow more time for planning tactics.

Several participants indicated cooperative tabletop games were more accessible than competitive games "because the sighted assistance is also there in the mix as well. So, it's not like it's all you, it's them and you" (P1). P6 and P8, however, shared that they do not mind games where player imbalance is a result of the design and not to "either my, lack of vision or the other players, visual ability that I find that to be less acceptable" (P6).

Finally, five participants suggested solutions to circumvent issues regarding cognitive load. P3 and P4 said tabletop games in which players are only responsible for their own information (vs. shared information) is simpler to manage. P7 and P13 similarly commented on their appreciation of games with games that are simple to memorize. In the case of $\mathrm{P} 7$, however, they informed us of the potential for balance between predictability and luck, "I like predictability, but there has to be a game of chance that goes with it like poker. You know exactly how it is going to go all the time, but there is a chance element." The proper design of tabletop game mechanics, with elements such as timed actions and concerns of cognitive load, proved to be an important accessibility consideration for BLV players.

4.3.3 Accessibility Considerations for Non-BLV Players. Seven participants placed a large focus on topics of inclusive design that considered all levels of sight. When discussing the importance of multiple modalities, P5 said, "a lot of players, most of the players, don't have any type of visual impairment, but they might be colour-blind so in this case. . the colour-blind player won't know the difference between red and green. But when they see, let's say a triangle for one and the circle for the other, they know."

In addition, participants defined accessible games that accounted the needs of those with non-vision disabilities. When discussing ergonomic controllers, P3 shared considerations for those with motor difficulties, "if you have mobility issues or hand issues, it's good to provide accessible controllers that are that won't pull you out of the gaming experience because you're uncomfortable." Despite initiated by a discussion of digital peripherals, concerns for motor difficulties are vital in tangible context due to physical manipulation. Similarly, P11 placed importance on providing accessibility for neurodivergence, "Say you were playing with someone who had some cognitive delays or disability or something you could adjust things around to see or depending on how people like it."

P3 and P10 described the concept of "universality of gaming" and said the process of adjusting game rules for specific players "add something on [the game] for everybody to play." P9 expressed, however, that the application of universal gaming principles to tabletop games did not always occur: "I understand that not all games are meant to be inclusive or even accessible, since you have games that are actually fully sight based. I would not be dissatisfied if I knew that there was no attempt by the developers of trying to make it accessible for games that. . As default they should attempt to make everything accessible as possible." Overall, it is crucial for people of varying levels of ability to equally participate in games, which makes the interaction between all players "a lot more fun" (P3).

4.3.4 Aesthetics versus Accessibility. Participants discussed the balance between game aesthetics and accessibility is an important consideration in the design of games for BLV. Seven participants expressed that the use of aesthetically pleasing and high-quality materials was vital in game design; these materials were crucial for BLV players who rely on the ability to physically manipulate the game elements. Superior materials made BLV players' games more durable and easier to transport without risk of damage. Comparably, participants reported that aesthetically pleasing sound lends itself to heightened immersion while gaming: "I have a good sense around audio, and I really like games that take advantage of that. With the lack of visuals, at least with audio, you can add that additional element of immersion that a lot of these games are sorely lacking” (P3).

Four participants, however, expressed that accessibility, not aesthetics, needs to be the utmost concern during game design. P8 arguably held the most vehement stance on accessibility over aesthetics, "if the game is terrible, no amount of good sound is going to matter." Their argument is particularly interesting as prior discussions highlighted the importance of sound use. The tension suggests that designers should focus on how their game uses sound 
functionally before examining the quality. Overall, participant insight highlights that, while accessibility is crucial, aesthetics must not be sacrificed lest impacting the tabletop game experience.

\subsection{Outcomes of Accessible Games}

We report on the outcomes of accessible games experienced by BLV players. First, accessible games allowed them to be independent to a certain degree. P10 described the importance of having independent gaming experiences: "and that's would say something that, you know. . . when you play, I think you should be able to do everything. And, you know, as independent as possible." While P8 described the independence in learning game rules, P1 and P3 emphasized the benefit of independence comes from the gameplay, particularly in no longer requiring the use of sighted assistance. P3 described it as an ease of burden: "So I didn't have to keep bothering them to ask what each card did in a turn on the table." They likewise said they appreciated the independence of "not have to constantly reach out and check what other people are doing right. So it's been a personal preference of mine."

Two participants also shared the impact that accessible games should have on the fairness of gameplay for players of varying levels of sight. When referring to the use of timed actions, P9 shared, "if the game was accessible, I would have no issues with it, since I would not have any troubles playing as fast or even faster than when someone who's sighted... so I would have an advantage even than someone sighted in certain scenarios so I would not be opposed to time-based games if it's if the game is well done." P10 elaborated on the use of timed actions as a measurement of fair gameplay: "it could have time. And I don't want to like, too easy, even if it's going to be something that requires competition and is going to have competition." This indicates that an accessible game would be able to have fair gameplay that could still maintain elements of time and competitiveness.

Finally, although completely beneficial in theory, four participants shared concerns with the enjoyment of completely accessible games. To be specific, three participants cautioned that different modalities of information communication are not always equal in their enjoyment. P14 expressed that audio games were not enjoyable and got boring very fast while P5 stated that "they cannot really be compared to actual mainstream video games." P3 echoed this sentiment by providing a comparison between mainstream First-Person Shooter games and audio games and how audio games take them out of the experience, "I was very much into first person shooter, so very hyper visual. And that's what really drew me in, it was the visuals of the game. But now everything is all done through audio, through text to speech engines. And some of the audio games are interesting and fun, but it's not the visual element which really kind of makes it more reminiscent of what I'm missing as opposed to keeping me involved in the game right now." This exemplifies that as important as it is to create accessible tabletop games, designers must keep the enjoyment of the game at the forefront.

\section{DISCUSSION}

Through a series of 15 semi-structured interviews, we explored tabletop gaming experiences and opinions of people who are BLV and observed four themes: (1) properties of inaccessible games, (2) outcomes of inaccessible games, (3) properties of accessible games, and (4) outcomes of accessible games. Our conversations regarding accessible tabletop game properties elicited rich ideation from participants to improve gaming accessibility. While participants had experience with all styles of tabletop game genres, the ideas participants generated ranged from tactile keys to communicate textured information or OCR-compatible rulebooks often being game-agnostic.

We designed this study with teachings from the social model of disability in mind. By placing the onus of inaccessibility on society and how the design of tabletop games has created barriers and how they should be designed better, our findings highlight how the BLV community is forced to navigate an environment that was not designed with their insight and expertise [21, 27, 66]. Our study therefore stresses the importance of taking on a Nothing About Us Without Us approach to create an accessible and enjoyable for individuals of any levels of sight without requiring the responsibility for adjustment to be placed upon them. [24, 93].

For researchers and designers who are new to the approach, they can consult the leading game industry's inclusive design principles. For instance, Microsoft stresses three principles: (1) recognize own biases, (2) learn from diversity by involving targeted users, and (3) solve for one, which can extend to many [59]. The goal is to arrive at solutions that benefit a targeted group and people universally. For instance, an accessible video-based rulebook with a closed captioning can benefit people with normal vision, such as children who may learn better via video than text. With this inclusive design thinking mindset, researchers and designers need to diverge from the existing trends in accessibility research that do not practice codesign with BLV participants and just play-test games instead [22, $46,54]$. Based on our findings, we leverage a deeper understanding of tabletop gaming accessibility to discuss design recommendations for researchers and game designers when creating tangible games that are both accessible and enjoyable for individuals of any levels of sight without requiring the responsibility for adjustment to be placed upon them.

Before exploring our design recommendations, it is necessary to reflect on the importance our work highlights on the need to better understand the current state of tabletop game interactivity for individuals who are BLV.

\subsection{Tabletop Game Interactivity for BLV Players}

Our participants shared a variety of insights that greatly inform the current state of tabletop game interactions for people who are BLV. One of the most prominent elements of interactivity for our participants is multimodality. What we demonstrate in this work, however, is that game designers often do not fully consider this interaction design. Interaction techniques such as the use of texture or sound are often omitted from the tabletop gaming experiences rather than being used as supplements to visual elements such as colour and text. Additionally, when tabletop games do include non-visual interactive elements, they are often not executed well such as creating physical game tokens that are not distinct or easily disturbed when feeling the board. 
Our work also highlights many shortcomings in the interactivity of many digital components of modern games. While many BLV players highlighted the usefulness of digital rulebooks as an accessible means of learning gameplay, many pointed out that these are often published incorrectly leading to incompatibility with popular assistive technologies like screen readers and OCR. Similar limitations arise from common barriers like the absence of sound cues or timed digital games like Trivia Crack not considering the use of screen readers. These limitations alone exemplify that, while often considered inherently accessibly to BLV players, digital components of tangible games also require thoughtful design (which we discuss further in the section 5.3.5).

Given what our work highlights regarding the interactivity of tabletop games and some of their digital components, it is vital for those pursuing interactive and accessible game design, both tangible and digital games alike, to include the BLV community in the entire process. It warrants future work to examine the process of tangible game design utilizing a practice of what Ladner [51] defines as design for user empowerment. A co-design process that applies a fully accessible lens to the standard design cycle of analyzing, designing, prototyping, and testing by involving end users, in this case the BLV community, at every stage. Once guidelines are developed, we encourage tangible game, and interaction designers in large, to implement these recommendations, regardless of game genre, as early in the design process as possible with further input received by BLV players regarding their implementation to ensure they are continuously reflecting the current needs of BLV players. This would better ensure that the interactive elements of tangible games are both accessible and engaging to the BLV community by giving BLV individuals more agency in the design of said games.

\subsection{Digitization of Tabletop Games}

We noted that recent tabletop game design research has largely focused on the creation of hybrid digital-tabletop gaming solutions $[46,65,81,83]$. We want to emphasize that while participants in this study provided an abundance of adaptation ideas, they all preserved the physical nature of tabletop games. Notable examples included adding the use of textures with a separate tactile key to distinguish game pieces or actively using Braille in addition to print text.

Adaptations that made use of technology were also presented to build upon the physical elements. NFC tags and QR codes are common ideas to serve as an additional layer of information over top of the visual aspects of tabletop games. OCR likewise appears as a popular of means of stripping down the visual barrier of tabletop games whenever applicable instead of being seen as an update to the game.

Considerations into the myriad of functions hybrid gaming models offer to players $[13,75,83]$ highlights the traction digital-tangible games have gained in the field of HCI as of late. Our study suggests the need for future accessible tangible gaming research to re-examine whether digitally based solutions are in fact appropriate, particularly when addressing accessibility concerns. Building of ability-based design practices [93], an in-depth examination into the acceptability of hybrid digital-tangible solutions would further contribute to a better understanding into what BLV individuals expect from their tangible gaming experiences.

\subsection{Design Guidelines for BLV Accessible Tabletop Games}

We compare our own guidelines against two design guidelines established by Tomé et al. [74] and Heron et al. [42]. We expand on some of their guidelines and we also offer new guidelines that center on game mechanics and aesthetics.

5.3.1 Accessible Rulebooks. Tomé et al. [74] emphasized on creating accessible rulebooks. We provide additional ideas on how designers can make accessible rulebooks. First, videos can become an effective alternative to text. While a standard practice for digital games, the tabletop game design has yet to popularize the use of video tutorials [2]. Since video instructions for tabletop games are only an emerging trend, discussion about tangible experiences often overlook the process of learning game rules. Considerations concerning the accessibility of tangible game rulebooks are necessary to ensure BLV players can access a game from the beginning. Also, game designers can consider utilizing mobile devices and emerging techniques such as QR codes and OCR to present instructions. As an example, a game community's website can have a scannable $\mathrm{QR}$ code and once a BLV player scans the code they would receive the game rules on their phone. Likewise, designers must remain up to date on the proper implementation of said technologies to avoid common issues such as uploading rulebooks as one single image that are not compatible with screen readers.

In addition, game designers should carefully consider how they write rulebooks. Game rulebooks often make use of images to present vital game information [74], particularly with games that necessitate the construction of components. Accessible rulebooks should present information in multiple forms, such as textures and descriptive audio. Just as accessible web content often uses the Web Content Accessibility Guidelines (WCAG) as a design reference, we advise designers to create a similar standardized guideline should exist for tangible game rulebooks to guide game designers.

5.3.2 Multimodality and Simplicity of Information. Accessible design literature commonly practices the use of non-visual means of information communication [23, 31, 74]. It is crucial to present game information along various dimensions. While Braille is often the default method of making game text accessible, it is no longer an effective accessibility measure on its own for two reasons: there is an increasing number of BLV individuals who do not receive Braille education [98] and sighted individuals can use Braille to gain an unfair advantage by memorizing patterns of others' cards. Game designers must carefully create non-visual elements to make sure they are usable by BLV participants. This is of importance when ensuring players do not knock over tangible elements when feeling the table for information or colours and fonts are distinguishable by those with low vision.

Tomé et al. [74] suggested utilizing audio to keep BLV players informed of game rules and changes on game state. By ensuring game information is multisensory whenever possible, game designers ensure BLV do not have to make additional adaptations to their games or purchase unique accessible versions. Tabletop game adaptations are often extremely costly, time-consuming, and difficult to undertake while accessible versions of games are often more expensive and nearly impossible to find should they exist. 
We further suggest that designers create game rules as simple as possible. Only limited amount of information should be communicated and understood by BLV players. This suggestion especially matters to time-based tabletop games that add pressure to and cause cognitive load in BLV players. When tabletop games involve timedbased actions, these actions need to be straightforward and games need to incorporate turn-based mechanisms to ease the pace (e.g., a skill to pause the game). Careful design consideration of game mechanics cannot be understated.

The MDA framework identifies three components that game designers need to consider, Mechanics, Dynamics, and Aesthetics [43]. Mechanics are game rules and components. Dynamics are players' response to rules and components. Aesthetics are the desired emotional responses in players. Applying the framework, in time-based tabletop games, for instance, providing one truth and four lies to an opponent under one minute is game mechanics and the resulting time pressure is dynamics. Together they can elicit negative emotions in conjunction with cognitive overload. If the goal of tabletop games is to elicit positive emotion in BLV players, game mechanics should be simple.

5.3.3 Encourage Autonomy in Games. When designing accessible tangible games, game designers must consider autonomous play for BLV players. Accessible tangible games should consider sighted assistance to be an extra feature to the game instead of a requirement; BLV individuals prefer playing with others instead of having others play for them. Tomé et al. [74] and Heron et al. [42] emphasised on designing tabletop games for autonomy through making sensory game elements accessible (i.e., text, sound, color, touch), the findings which we have also replicated. We further suggest that designers empower BLV players of different levels of sight with the capacity to customize game mechanics. The ability to adjust a variety of settings is a common digital game design trend that tangible games can easily adopt. By providing players the ability to fine-tune games to meet their needs, tangible games provide unique and inclusive game experiences for different disabilities.

Tabletop games must be compatible with assistive technologies that BLV players use. Conversations regarding the design rulebooks arise when discussing autonomy in tabletop games. While learning game rules is a collaborative experience amongst players, sighted assistance should not be a requirement for BLV players to learn game mechanics. Game rulebooks should not be saved as PDF images or written in columns; OCR and a screen reader work best with game rulebooks written in a single column in Microsoft Word [14]. Any icons or visual images should be accompanied by alt-text to be read with a screen reader.

When it comes to tabletop game contents, designers need to consider how a combination of assistive technologies can work in harmony. Many of our participants used a screen reader. However, the extent to which a screen reader itself can assist BLV players in gameplaying is limited (e.g., it cannot not read symbols on cards). Hence, designers should think about how a screen reader can be used in combination with emerging technologies and mobile devices (e.g., QR codes or NFC tags, as discussed previously).

5.3.4 Practicality of Aesthetics. When proactively designing a tangible game with accessibility in mind, it is important to consider the game's aesthetic elements as well. Aesthetically pleasing visual elements have been shown to be an important factor in improving the enjoyment of tabletop gaming for BLV players [74]. We argue that high quality materials are also important for ensuring that BLV players who rely on touch are still able to feel immersed within the gaming experience. They are likewise important when ensuring the durability and transportation of tangible games. There are two noteworthy points regards to this point. First, this line of finding extends on the MDA framework [43], which outlined sensation/pleasure as one of the aesthetics goals. While the framework stated game mechanics and dynamics as the main trigger of aesthetics goals, our finding suggests the materiality of game components can contribute to aesthetics goals. Second, while Tomé et al. [74] and Heron et al. [42] conceptualize touch in terms of tactile distinction of game pieces, we expand on this conceptualization to incorporate sense-pleasure of game pieces.

5.3.5 Design Considerations for Tangible Games. It is important to highlight that our guidelines also contribute towards improving the accessibility of tangible games. In tangible games, players interact with a digital game environment by physically moving and manipulating tangible objects $[8,53]$. As an example, in the game called WeatherGods [8] a player moves three tangible objects-a camel rider, a detector, a bandit-on a digital tabletop and every aspect of game content are displayed on the table.

Touch, sound, and visual cues are still the main communication cues for tangible games and our design guidelines apply to the digital and physical components of tangible games. First, BLV players must be able to distinguish between tangible play objects based on touch. Tangible play objects tend to be in a big size. For instance, chess-like game pieces in Bakker and Vorstenbosch [8] and farm animal pieces in Marco and Cerezo [53] come in the size of a human fist. Also, materials that make up tangible objects must be aesthetically pleasing to elicit sensation and pleasure in BLV players.

Second, game information displaced on a digital tabletop should use good color contrast and a large font size. With tangible games, BLV players should be able to configure color contrast and font size themselves, just as they would with configuring settings in regular computers. This game setting customization can further increase their sense of autonomy. Third, ensuring the simplicity of game mechanics and time-based actions is important against the possibility of increased cognitive load as BLV players interact with physical and digital elements in tangible games. One way to achieve this is by including game mechanics that allow BLV players to take a step back at any point during the gameplay.

Lastly, provide BLV players with accessible game rulebooks displayed on a digital tabletop and rulebooks can be manipulated through tangible objects. BLV players can scroll through rulebooks using an object in a similar manner as they would with a mouse on computers. Designers can make this learning of game rulebooks more entertaining by having a tangible object to represent a player's virtual avatar on a digital game environment and have their virtualself to guide them through rulebooks.

\subsection{Limitations and Future Work}

There are several caveats in our study. First, while we had a large number of participants compared to other similar works $[3,4,74]$, 
we recognize the number is still somewhat limited. Second, we had a small number of participants who exclusively played video games, although they had played board and card games in past. Some of them could not elaborate on their experiences with tabletop games, which reduced the overall richness in our data.

Relatedly to external validity of our findings, our participants' tabletop gaming experiences centered on eight game genres. Yet these genres are the most popular genres and the overall makeup of tabletop games should be comparable across genres (e.g., physical and social set-up, interaction with game pieces). We remain somewhat confident that other researchers can use our findings to make a valid inference to BLV players' tabletop gaming experience with other genres.

Third, much of our conversations with participants focused on what makes tabletop games accessible, a factor we recognize is only a subset of the overall tabletop gaming experience. Future iterations on similar investigations should explore the different tabletop game elements that BLV players enjoy from a purely entertainment perspective.

Fourth, we acknowledge the potential limitation of our binary theme structure in interpreting our findings. It implies inaccessible tabletop games only consist of "bad" game properties and outcomes while accessible tabletop games consist of only "good" game properties and outcomes. Some of these properties and outcomes may exist as a continuum for (in)accessible tabletop games. Inaccessible games can foster dependency and independency, just as accessible tabletop games can result in fair and unfair play. Some properties that make tabletop games inaccessible (e.g., poor color contrast) can exist in accessible tabletop games. However, it likely that a BLV player experiences negative aspects of tabletop games at a greater frequency than positive aspects. The opposite is likely for accessible tabletop games.

Despite these caveats, our study introduces exciting future work. There is an opportunity to test the applications of these tangible accessible dimensions in a co-design workshop with the BLV individuals, which would not only test the feasibility of these dimensions but also ensure creating an accessible game that truly accounts the needs of BLV players.

Secondly, existing game accessibility research has yet to explore the learning of rules for BLV players and, as such, an opportunity is presented to explore accessible solutions and adaptations for game instructions.

Finally, several participants discussed the pros and cons of using AI devices to facilitate tangible gameplay. Making use of this knowledge, it would be worthwhile to perform a user-centered, iterative design study of AI solutions that would aid BLV users through the entire process of tangible gameplay. Observations of gameplay sessions in this type of study may even be able to lend themselves to further understanding the gaming practices of those who are BLV.

\section{CONCLUSION}

We explored the experiences of BLV individuals with tabletop gaming and found four common themes that highlight properties of (in)accessible games and their outcomes. BLV players' experiences demonstrate how elements such as colour, touch, text, and game mechanics act simultaneously as barriers and facilitators to tabletop game accessibility. Furthermore, these experiences exemplify how these elements influence processes of adaptation, acclimatization, and inclusive design. We also discuss brief design recommendations for accessible tangible games along with consideration into accessible rulebooks, multimodality, autonomy, and aesthetics. We conclude by emphasizing the importance for future work to consider the validity of pursuing digitization of tabletop games for accessibility.

Our work provides an in-depth examination into the tabletop gaming backgrounds of BLV people, and the impact accessibility has on their board and card gaming practices. Our study advances research on accessible tangible games by providing a recent insight into the importance of understanding the needs and desires of tabletop game players with different levels of ability. By leveraging the lessons from these first-hand accounts of what makes a tabletop game (in)accessible, game designers will be able to create tangible games that BLV and sighted individuals can both enjoy.

\section{ACKNOWLEDGMENTS}

This work was supported and funded by the National Sciences and Engineering Research Council of Canada (NSERC) through a Discovery grant (2017-06300), a Discovery Accelerator Supplement (2017-507935), and the Collaborative Learning in Usability Experiences CREATE grant (2015-465639). We would like to thank our participants for their time, expertise, and insights.

\section{REFERENCES}

[1] Lúcia Abreu, Ana Cristina Pires, and Tiago Guerreiro. 2020. TACTOPI: A Playful Approach to Promote Computational Thinking for Visually Impaired Children. In Proceedings of the 22nd International ACM SIGACCESS Conference on Computers and Accessibility (ASSETS '20), October 26-28, 2020, Virtual Event, Greece. ACM Inc., New York, NY, 1-3. DOI:https://doi.org/10.1145/3373625.3418003

[2] Erik Andersen, Eleanor O'rourke, Yun-En Liu, Richard Snider, Jeff Lowdermilk, David Truong, Seth Cooper, and Zoran Popovic. 2012. The Impact of Tutorials on Games of Varying Complexity. In Proceedings of the SIGCHI Conference on Human Factors in Computing Systems (CHI '12), May 5-10, 2012, Austin, Texas. ACM Inc., New York, NY, 59-68

[3] Ronny Andrade, Melissa J. Rogerson, Jenny Waycott, Steven Baker, and Frank Vetere. 2019. Playing blind: Revealing the world of gamers with visual impairment. In Proceedings of the Conference on Human Factors in Computing Systems, May 4-9, 2019, Glasgow, Scotland. ACM Inc., New York, NY, 1-14. DOI:https: //doi.org/10.1145/3290605.3300346

[4] Ronny Andrade, Melissa J Rogerson, Jenny Waycott, Steven Baker, and Frank Vetere. 2020. Introducing the Gamer Information-Control Framework: Enabling Access to Digital Games for People with Visual Impairment. In Proceedings of the Conference on Human Factors in Computing Systems, April 25-30, 2020, Honolulu, HI. ACM Inc., New York, NY, 1-14. DOI:https://doi.org/10.1145/3313831. 3376211

[5] Alissa N. Antle. 2007. The CTI framework: Informing the design of tangible systems for children. In Proceedings of the 1st International Conference on Tangible and Embedded Interaction (TEI'07), February 15-17, 2007, Baton Rouge, Louisiana. ACM Inc., New York, NY, 15-17. DOI:https://doi.org/10.1145/1226969. 1227010

[6] Dominique Archambault and Damien Olivier. 2005. How to Make Games for Visually Impaired Children. In Proceedings of the 2005 ACM SIGCHI International Conference on Advances in Computer Entertainment Technology (ACE '05), Valencia, Spain. ACM Inc., New York, NY, 450-453. DOI:https://doi.org/https: //doi.org/10.1145/1178477.1178578

[7] Matthew T Atkinson, Sabahattin Gucukoglu, Colin H C Machin, and Adrian E Lawrence. 2006. Making the Mainstream Accessible: Redefining the Game The AGRIP Project. In Proceedings of the 2006 ACM SIGGRAPH Symposium on Videogames (Sandbox '06), July 29-30, 2006, Boston, Massachusetts. ACM Inc., New York, NY, 21-28. DOI:https://doi.org/https://doi.org/10.1145/1183316.1183321

[8] Saskia Bakker, Debby Vorstenbosch, Elise Van Den Hoven, Gerard Hollemans, and Tom Bergman. 2007. Weathergods: Tangible interaction in a digital tabletop game. In Proceedings of the 1st International Conference on Tangible and Embedded 
Interaction (TEI'07), February 15-17, 2007, Baton Rouge, Louisiana. ACM Inc., New York, NY, 151-152. DOI:https://doi.org/10.1145/1226969.1227000

[9] Mark Barlet and Steve Spohn. 2012. A Practical Guide to Game Accessibility. Retrieved April 8, 2021 from https://accessible.games/wp-content/uploads/2018/ 11/AbleGamers_Includification.pdf

[10] Erin E. Barton, Elizabeth A. Pokorski, Erin M. Sweeney, Marina Velez, Stephanie Gossett, Jia Qiu, Celia Flaherty, and Maddisen Domingo. 2018. An Empirical Examination of Effective Practices for Teaching Board Game Play to Young Children. J. Posit. Behav. Interv. 20, 3 (July 2018), 138-148. DOI:https://doi.org/ $10.1177 / 1098300717753833$

[11] Jen Beeston, Christopher Power, Paul Cairns, and Mark Barlet. 2018. Accessible player experiences (APX): The players. In Lecture Notes in Computer Science (including subseries Lecture Notes in Artificial Intelligence and Lecture Notes in Bioinformatics), Springer Verlag, 245-253. DOI:https://doi.org/10.1007/978-3319-94277-3 40

[12] Srinjita Bhaduri, Jesús G Ortiz Tovar, and Shaun K Kane. 2017. Fabrication Games: Using 3D Printers to Explore New Interactions for Tabletop Games. In Proceedings of the 2017 ACM SIGCHI Conference on Creativity and Cognition (C\&C '17), June 27-30, 2017, Singapore, Singapore. ACM Inc., New York, NY, 51-62. DOI:https://doi.org/10.1145/3059454.3059463

[13] Arpit Bhatia and Rakshita Anand. 2021. An Analysis of Ludo Board Game Play on Smartphones; An Analysis of Ludo Board Game Play on Smartphones. In Proceedings of the $2021 \mathrm{CHI}$ Conference on Human Factors in Computing Systems (CHI EA'21), May 8-13, 2021, Yokohama, Japan. ACM Inc., New York, NY, 1-6. DOI:https://doi.org/10.1145/3411763.3451728

[14] BoardGameGeek. Blind gamer searching for accessible rulebook. 2019

[15] Pranjal Protim Borah. 2020. Deformation Gesture-based Input Method for Nonvisual Primitive Geometric Shape Drawing. In Proceedings of the 14th International Conference on Tangible, Embedded, and Embodied Interaction (TEI '20), February 9-12, 2020, Sydney, NSW, Australia. ACM Inc., New York, NY, 911-915. DOI:https://doi.org/10.1145/3374920.3374960

[16] Pranjal Protim Borah, Ayaskant Panigrahi, and Keyur Sorathia. 2020. TMOVE: Multimodal Feedback Actuator for Non-visual Exploration of Virtual Lines. In Proceedings of the 14th International Conference on Tangible, Embedded, and Embodied Interaction (TEI '20), February 9-12, 2020, Sydney, NSW, Australia. ACM Inc., New York, NY, 603-610. DOI:https://doi.org/10.1145/3374920.3374994

[17] Mollie Braley, Yifei Zhao, Nisha Kunhikrishnan, Matthew Bofenkamp, Siyu Chen, Michael Christel, Yu-Kai Chiu, and Jessica Hammer. 2019. Promoting Player Empathy for People Living with Poverty. In Proceedings of the Annual Symposium on Computer-Human Interaction in Play Companion (CHI PLAY EA'19), October 22-25, 2019, Barcelona, Spain. ACM Inc., New York, NY, 233-239. DOI:https://doi.org/10.1145/3341215.3358244

[18] Virginia Braun and Victoria Clarke. 2006. Using thematic analysis in psychology. Qual. Res. Psychol. 3, 2 (January 2006), 77-101. DOI:https://doi.org/10.1191/ 1478088706qp063oa

[19] Virginia Braun, Victoria Clarke, and Gareth Terry. 2014. Using thematic analysis in psychology. In Qualitative Research in Clinical and Health Psychology. 95-113. DOI:https://doi.org/10.1007/978-1-137-29105-9 7

[20] Daniella Briotto Faustino, Sara Nabil, and Audrey Girouard. 2020. Bend or PIN: Studying Bend Password Authentication with People with Vision Impairment. In Proceedings of Graphics Interface Conference 2020, May 28-29, 2020, Toronto, Canada. 183-191. DOI:https://doi.org/10.20380/GI2020.19

[21] Tania Burchardt. 2010. Capabilities and disability: the capabilities framework and the social model of disability. Disabil. Soc. 19,7 (October 2010), 753-751. DOI:https://doi.org/10.1080/0968759042000284213

[22] Matthew Butler, Leona Holloway, Samuel Reinders, Cagatay Goncu, and Kim Marriott. 2021. Technology Developments in Touch-Based Accessible Graphics: A Systematic Review of Research. In Proceedings of the $2021 \mathrm{CHI}$ Conference on Human Factors in Computing Systems, May 8-13, 2021, Yokohama, Japan. ACM Inc., New York, NY, 1-15. DOI:https://doi.org/10.1145/3411764.3445207

[23] Paul Cairns, Christopher Power, Mark Barlet, and Greg Haynes. 2019. Future design of accessibility in games: A design vocabulary. Int. J. Hum. Comput. Stud. 131, (November 2019), 64-71. DOI:https://doi.org/10.1016/j.ijhcs.2019.06.010

[24] James Charlton. 1998. Nothing About Us Without Us: Disability Oppression and Empowerment. University of California Press. DOI:https://doi.org/10.1525/ california/9780520207950.001.0001

[25] Wing Chin. 2015. Around 92\$ of people with impairments play games despite difficulties. Retrived from https://www.game-accessibility.com/documentation/ around-92-of-people-with-impairments-play-games-despite-difficulties/

[26] Aishat Aloba, Gabriel Coleman, Triton Ong, Shan Yan, Dehlia Albrecht, Marko Suvajdzic, and Lisa Anthony. 2017. From Board Game to Digital Game: Designing a Mobile Game for Children to Learn About Invasive Species. In Extended Abstracts Publication of the Annual Symposium on Computer-Human Interaction in Play (CHI PLAY EA'17), October 15-18, 2017, Amsterdam, The Netherlands. ACM Inc., New York, NY, 375-382. DOI:https://doi.org/10.1145/3130859.3131326

[27] Hazel Dixon. 2021. Immersive Performance and Inclusion Through a Lens of the Social Model of Disability. Interactions (2021), 70-72. DOI:https://doi.org/10. $1145 / 3334480.3375150$
[28] Konstantinos Drossos, Nikolaos Zormpas, Andreas Floros, and George Giannakopoulos. 2015. Accessible games for blind children, empowered by binaural sound. In Proceedings of the 8th ACM International Conference on Pervasive Technologies Related to Assistive Environments (PETRA 2015), July 1-3, 2015, Corfu, Greece. ACM Inc., New York, NY, 1-8. DOI:https://doi.org/10.1145/2769493. 2769546

[29] Owen Duffy. 2014. Board games' golden age: sociable, brilliant, and driven by the Internet. The Guardian. Retrieved from https://www.theguardian.com/ technology/2014/nov/25/board-games-internet-playstation-xbox

[30] Yasmine N. El-Glaly, Francis Quek, Tonya Smith-Jackson, and Gurjot Dhillon. 2013. Touch-Screens are Not Tangible: Fusing Tangible Interaction with Touch Glass in Readers for the Blind. In Proceedings of the 7th International Conference on Tangible, Embedded and Embodied Interaction (TEI '13), February 10-13, 2013, Barcelona, Spain. ACM Inc., New York, NY, 245-253. DOI:https://doi.org/https: //doi.org/10.1145/2460625.2460665

[31] Barrie Ellis, Gareth Ford-Williams, Lynsey Graham, Dimitris Grammenos, Ian Hamilton, Ed Lee, Jake Manion, and Thomas Westin. Games accessibility guidelines. Retrieved from http://gameaccessibilityguidelines.com/

[32] Malin Eriksson, Ben Kenward, Leo Poom, and Gunilla Stenberg. 2021. The behavioral effects of cooperative and competitive board games in preschoolers. Scand. J. Psychol. (2021). DOI:https://doi.org/10.1111/sjop.12708

[33] Matthew Ernst, Travis Swan, Victor Cheung, and Audrey Girouard. 2017. Typhlex: Exploring Deformable Input for Blind Users Controlling a Mobile Screen Reader. Pervasive Comput. (2017), 28-35. Retrieved July 7, 2021 from www.apple.com/ca

[34] Vinitha Gadiraju, Annika Muehlbradt, and Shaun K. Kane. 2020. BrailleBlocks: Computational Braille Toys for Collaborative Learning. In Proceedings of the 21st International ACM SIGACCESS Conference on Computers and Accessibility (ASSETS '19), April 25-30, 2020, Honolulu, HI. ACM Inc., New York, NY, 1-12. DOI:https://doi.org/10.1145/3313831.3376295

[35] Barney Glaser and Anselm Strauss. 1967. The Discovery of Grounded Theory: Strategies for Qualitative Research. AldineTransaction.

[36] Ulrike Gollner, Tom Bieling, and Gesche Joost. 2012. Mobile Lorm Glove: Introducing a Communication Device for Deaf-Blind People. In Proceedings of the 6th International Conference on Tangible, Embedded and Embodied Interaction (TEI '12), February 19-22, 2012, Kingston, ON, Canada. ACM Inc., New York, NY, 127-130. DOI:https://doi.org/https://doi.org/10.1145/2148131.2148159

[37] David Gonçalves and André Rodrigues. 2020. Playing With Others: Depicting Multiplayer Gaming Experiences of People With Visual Impairments. In Proceedings of the 22nd International ACM SIGACCESS Conference on Computers and Accessibility (ASSETS '20), October 26-28, 2020, Virtual Event, Greece. ACM Inc., New York, NY, 1-12. DOI:https://doi.org/10.1145/3373625.3418304

[38] Dimitris Grammenos, Anthony Savidis, Yannis Georgalls, and Constantine stephanidis. 2006. Access Invaders: Developing a Universally Accessible Action Game. In Computers Helping People with Special Needs, Springer, 388-395.

[39] Hamilton A. Hernandez, T.C. Nicholas Graham, Darcy Fehlings, Lauren Switzer, Zi Ye, Quentin Bellay, Md Ameer Hamza, Cheryl Savery, and Tadeusz Stach. 2012. Design of an Exergaming Station for Children with Cerebral Palsy. In Proceedings of the SIGCHI Conference on Human Factors in Computing Systems, May 5-10, 2012, Austin, Texas. ACM Inc., New York, NY, 2619-2628. DOI:https: //doi.org/10.1145/2207676.2208652

[40] Hamilton A. Hernandez, Zi Ye, T.C. Nicholas Graham, Darcy Fehlings, and Lauren Switzer. 2013. Designing Action-Based Exergames for Children with Cerebral Palsy. In Proceedings of the SIGCHI Conference on Human Factors in Computing Systems, April 27-May 2, 2013, Paris, France. ACM Inc., New York, NY, 1261-1270. DOI:https://doi.org/https://doi.org/10.1145/2470654.2466164

[41] Michael James Heron, Pauline Helen Belford, Hayley Reid, and Michael Crabb. 2018. Eighteen Months of Meeple Like Us: An Exploration into the State of Board Game Accessibility. Comput. Games J. 7, 2 (June 2018), 75-95. DOI:https: //doi.org/10.1007/s40869-018-0056-9

[42] Michael James Heron, Pauline Helen Belford, Hayley Reid, and Michael Crabb. 2018. Meeple Centred Design: A Heuristic Toolkit for Evaluating the Accessibility of Tabletop Games. Comput. Games J. 7, 2 (June 2018), 97-114. DOI:https://doi. org/10.1007/s40869-018-0057-8

[43] Robin Hunicke, Marc Leblanc, and Robert Zubek. 2004. MDA: A Formal Approach to Game Design and Game Research. In Proceedings of the AAAI Workshop on Challenges in Game AI, 1722

[44] IGDA-GASIG. 2015. SIG Top Ten. Retrieved from https://igda- gasig.org/how/sigtop-ten/

[45] Michael Ion, Dimitris Sacharidis, and Hannes Werthner. 2020. Designing a Recommender System for Board Games. In Proceedings of the 35thAnnual ACM Symposium on Applied Computing (SAC '20), March 30-April 3, 2020, Brno, Czech Republic. ACM Inc., New York, NY, 1465-1467. DOI:https://doi.org/10. $1145 / 3341105.3375780$

[46] Gabriella M. Johnson and Shaun K. Kane. 2020. Game Changer: Accessible Audio and Tactile Guidance for Board and Card Games. In Proceedings of the 17th International Web for All Conference, April 20-21, 2020, Taipei, Taiwan. New York, NY, USA, ACM Inc., New York, NY, 1-12. DOI:https://doi.org/10.1145/ 3371300.3383347 
[47] Claire Kearney-Volpe, Amy Hurst, and Scott Fitzgerald. 2019. Blind Web Development Training at Oysters and Pearls Technology Camp in Uganda. In Proceedings of the 16th International Web for All Conference (W4A '19), May 13-15, 2019, San Francisco, California. ACM Inc., New York, NY, 1-10. DOI:https: //doi.org/10.1145/3315002.3317562

[48] Imran Khaliq and Isabelle Dela Torre. 2019. A Study on Accessibility in Games for the Visually Impaired. In Proceedings of the EAI International Conference on Smart Objects and Technologies for Social Good (GoodTechs '19), September 25-27, 2019, Valencia, Spain, ACM Inc., New York, NY, 142-148. DOI:https://doi. org/https://doi.org/10.1145/3342428.3342682

[49] Shannon Knights, Nicholas Graham, Lauren Switzer, Hamilton Hernandez, Zi Ye, Briar Findlay, Wen Yan Xie, Virginia Wright, and Darcy Fehlings. 2014. An innovative cycling exergame to promote cardiovascular fitness in youth with cerebral palsy. Dev. Neurorehabil. 19, 2 (2014), 135-140. DOI:https://doi.org/10 3109/17518423.2014.923056

[50] Varsha Koushik, Darren Guinness, and Shaun K Kane. 2019. StoryBlocks: A Tangible Programming Game to Create Accessible Audio Stories. In Proceedings of the 2019 CHI Conference on Human Factors in Computing Systems, May 4-9, 2019, Glasgow, Scotland, UK. ACM Inc., New York, NY, 1-12. DOI:https: //doi.org/10.1145/3290605.3300722

[51] Richard Ladner. 2015. Design for Empowerment. Interactions 22, 2 (2015), 24-29. DOI:https://doi.org/10.1145/2723869

[52] Sebastián Aced López, Fulvio Corno, and Luigi De Russis. 2015. Can we make dynamic, accessible and fun one-switch video games? In Proceedings of the 17th International ACM SIGACCESS Conference on Computers and Accessibility (ASSETS'15), October 26-28, 2015, Lisbon, Portugal. ACM Inc., New York, NY, 421-422. DOI:https://doi.org/10.1145/2700648.2811333

[53] Javier Marco, Eva Cerezo, and Sandra Baldassarri. 2010. Playing with toys on a tabletop active surface. In Proceedings of IDC2010: The 9th International Conference on Interaction Design and Children. DOI:https://doi.org/10.1145/1810543. 1810596

[54] Monalize Ribeiro Mariano, Cristiana Brasil de Almeida Rebouças, and Lorita Marlena Freitag Pagliuca. 2013. Educative game on drugs for blind individuals: development and assesment. J. USP Sch. Nurs. 47, 4 (2013), 930-936. DOI:https: //doi.org/10.1590/S0080-623420130000400022

[55] Tiago Marques, Francisco Nunes, Paula Silva, and Rui Rodrigues. 2011. Tangible interaction on tabletops for elderly people. In Lecture Notes in Computer Science (including subseries Lecture Notes in Artificial Intelligence and Lecture Notes in Bioinformatics). DOI:https://doi.org/10.1007/978-3-642-24500-8_61

[56] Rachel Menzies. 2019. Unlocking Accessible Escape Rooms. In Proceedings of the 21st International ACM SIGACCESS Conference on Computers and Accessibility (ASSETS '19), October 28-30, 2019, Pittsburgh, Pennsylvania. ACM Inc., New York, NY, 510-512. DOI:https://doi.org/10.1145/3308561.3354611

[57] Fernando de Mesentier Silva, Scoo Lee, Julian Togelius, and Andy Nealen. 2017. AI-based Playtesting of Contemporary Board Games. In Proceedings of the 12th International Conference on the Foundations of Digital Games (FDG '17) August 14-17, 2017, Hyannis, Massachusetts. ACM Inc., New York, NY, 1-10. DOI:https://doi.org/10.1145/3102071.3102105

[58] Oussama Metatla, Sandra Bardot, Clare Cullen, Marcos Serrano, and Christophe Jouffrais. 2020. Robots for Inclusive Play: Co-designing an Educational Game With Visually Impaired and Sighted Children. In Proceedings of the Conference on Human Factors in Computing Systems, April 25-30, 2020, Honolulu, HI. ACM Inc., New York, NY, 1-13. DOI:https://doi.org/10.1145/3313831.3376270

[59] Microsoft. Inclusive Design. Retrived from https://www.microsoft.com/design/ inclusive/

[60] Daniel Miller, Aaron Parecki, and Sarah A Douglas. 2007. Finger Dance: A sound game for blind people. In Proceedings of the 9th International ACM SIGACCESS Conference on Computers and Accessibility (ASSETS '07), October 14-17, 2007, Tempe, Arizona. ACM Inc., New York, NY, 253-254. DOI:https://doi.org/https: //doi.org/10.1145/1296843.1296898

[61] Tony Morelli, John Foley, Luis Columna, Lauren Lieberman, Suny Brockport, and Eelke Folmer. 2010. VI-Tennis: a Vibrotactile/Audio Exergame for Players who are Visually Impaired. In Proceedings of the 5th International Conference on the Foundations of Digital Games (FDG '10), June 19-21, 2010, Monterey, California, ACM Inc., New York, NY, 147-154. DOI:https://doi.org/https://doi.org/10.1145/ 1822348.1822368

[62] Tony Morelli, John Foley, and Eelke Folmer. 2010. VI-Bowling: A Tactile Spatial Exergame for Individuals with Visual Impairments. In Proceedings of the 12th International ACM SIGACCESS Conference on Computers and Accessibility (ASSETS '10), October 25-27, 2010, Orlando, Florida. ACM Inc., New York, NY, 179-186. DOI:https://doi.org/https://doi.org/10.1145/1878803.1878836

[63] Tony Morelli, John Foley, Lauren Lieberman, and Eelke Folmer. 2011. Pet-NPunch: Upper Body Tactile/Audio Exergame to Engage Children with Visual Impairments into Physical Activity. In Proceedings of Graphics Interface Conference, May 25-27, 2011, St. John's, Newfoundland, Canada. 223-230. DOI:https //doi.org/10.5555/1992917.1992954

[64] Tony Morelli and Eelke Folmer. 2011. Real-time Sensory Substitution to Enable Players who are Blind to Play Video games using Whole Body Gestures. In
Proceedings of the 6th International Conference on Foundations of Digital Games (FDG '11), June 29-July 1, 2011, Bordeaux, France. ACM Inc., New York, NY, 147153. DOI:https://doi.org/https://doi.org/10.1145/2159365.2159385

[65] Kirsty Noble and Michael Crabb. 2016. Projection Mapping As a Method to Improve Board Game Accessibility. SIGACCESS Accessibility and Computing. DOI:https://doi.org/10.5555/1992917.1992954

[66] Mike Oliver. 2013. The social model of disability: thirty years on. Disabil. Soc. 28, 7 (July 2013), 1024-1026. DOI:https://doi.org/10.1080/09687599.2013.818773

[67] Kaitlyn Ouverson. Accessible game design: the Domino effect. UX Collective. Retrieved April 8, 2021 from https://uxdesign.cc/accessible-game-design-thedomino-effect-55b8464779a

[68] Ana Cristina Pires, Filipa Rocha, Antonio José De Barros Neto, Hugo Simão, Hugo Nicolau, and Tiago Guerreiro. 2020. Exploring Accessible Programming with Educators and Visually Impaired Children. In Proceedings of the Interaction Design and Children Conference (IDC '20), June 21-24, 2020, London, UK. ACM Inc., New York, NY, 148-160. DOI:https://doi.org/10.1145/3392063.3394437

[69] PopCap Games. 2008. PopCap Games Research.

[70] John R Porter. 2014. Understanding and Addressing Real-World Accessibility Issues in Mainstream Video Games. SIGACCESS Accessibility and Computing 108. DOI:https://doi.org/https://doi.org/10.1145/2591357.2591364

[71] Shi Qiu, Siti Aisyah Anas, Hirotaka Osawa, Matthias Rauterberg, and Jun Hu. 2016. E-Gaze Glasses: Simulating Natural Gazes for Blind People Work-in-Progress. In Proceedings of the 10th International Conference on Tangible, Embedded, and Embodied Interaction (TEI '16), February 14-17, 2016, Eindhoven, Netherlands. ACM Inc., New York, NY, 563-569. DOI:https://doi.org/10.1145/2839462.2856518

[72] Kyle Rector, Cynthia L Bennett, and Julie A Kientz. 2013. Eyes-Free Yoga: An Exergame Using Depth Cameras for Blind \& Low Vision Exercise. In Proceedings of the 15th International ACM SIGACCESS Conference on Computers and Accessibility (ASSETS '13), October 21-23, 2013, Bellevue, Washington. ACM Inc., New York, NY, 1-8. DOI:https://doi.org/https://doi.org/10.1145/2513383.2513392

[73] Georg Regal, David Sellitsch, Simone Kriglstein, Simon Kollienz, and Manfred Tscheligi. 2020. Be Active! Participatory Design of Accessible Movement-Based Games. In Proceedings of the 14th International Conference on Tangible, Embedded, and Embodied Interaction (TEI '20), February 9-12, 2020, Sydney, NSW, Australia. ACM Inc., New York, NY, 179-192. DOI:https://doi.org/10.1145/3374920. 3374953

[74] Frederico Da Rocha Tomé Filho, Pejman Mirza-Babaei, Bill Kapralos, and Glaudiney Moreira Mendonça. 2019. Let's Play Together: Adaptation guidelines of board games for players with visual impairment. In Proceedings of the Conference on Human Factors in Computing Systems, May 4-9, 2019, Glasgow, Scotland. ACM Inc., New York, NY, 1-15. DOI:https://doi.org/10.1145/3290605.3300861

[75] Melissa J Rogerson and Lucy A Sparrow. 2021. Unpacking "Boardgames with Apps": The Hybrid Digital Boardgame Model." In Proceedings of the $2021 \mathrm{CHI}$ Conference on Human Factors in Computing Systems, May 8-13, 2021, Yokohama, Japan, 17. ACM Inc., New York, NY, 1-17. DOI:https://doi.org/10.1145/3411764. 3445077

[76] Patrick Roth, Lori Petrucci, and Thierry Pun. From Dots To Shapes: an auditory haptic game platform for teaching geometry to blind pupils.

[77] Luis Salvador-Ullauri, Angel Jaramillo-Alcázar, and Sergio Luján-Mora. 2017. A serious game accessible to people with visual impairments. In ACM International Conference Proceeding Series, ACM, 84-88. DOI:https://doi.org/10.1145/3175536. 3175576

[78] Jaime Sánchez, Nelson Baloian, Tiago Hassler, and Ulrich Hoppe. 2003. AudioBattleship: Blind Learners Collaboration through Sound. In Proceedings of the Conference on Human Factors in Computing Systems (CHI EA'03), April 5-10, 2003, Ft. Lauderdale, Florida. ACM Inc., New York, NY, 798-799. DOI:https://doi.org/https://doi.org/10.1145/765891.765998

[79] Jaime Sánchez, Mauricio Saenz, and Jose Miguel Garrido. 2010. Usability of a Multimodal Video Game to Improve Navigation Skills for Blind Children. ACM Trans. Access. Comput. 3, 7 (2010). DOI:https://doi.org/10.1145/1857920.1857924

[80] Ashrith Shetty, Ebrima Jarjue, and Huaishu Peng. 2020. Tangible Web Layout Design for Blind and Visually Impaired People: An Initial Investigation. In Adjunct Publication of the 33rd Annual ACM Symposium on User Interface Software and Technology, October 20-23, Virtual Event, USA. ACM Inc., New York, NY, 37-39. DOI:https://doi.org/10.1145/3379350.3416178

[81] Aditi Singh, Joanie Ouellet, and Victor Cheung. 2019. Re-Twist: Evaluating engagement in a digitally augmented traditional game. In Proceedings of the 13th International Conference on Tangible, Embedded, and Embodied Interaction (TEI'19),March 17-20, 2019, Temple, Arizona. ACM Inc., New York, NY, 181-187. DOI:https://doi.org/10.1145/3294109.3300976

[82] Manohar Swaminathan, Sujeath Pareddy, Tanuja Sawant, and Shubi Agarwal. 2018. Video Gaming for the Vision Impaired. In Proceedings of the 20th International ACM SIGACCESS Conference on Computers and Accessibility, October 22-24, 2018, Galway, Ireland. ACM Inc., New York, NY, 465-467. DOI:https: //doi.org/10.1145/3234695.3241025

[83] Lauren Thevin, Nicolas Rodier, Bernard Oriola, Martin Hachet, Christophe Jouffrais, and Anke M Brock. 2021. Inclusive Adaptation of Existing Board Games for Gamers with and without Visual Impairments using a Spatial Augmented Reality 
Framework for Touch Detection and Audio Feedback Games for Gamers with and without Visual Impairments using a Spatial Augmented Reality Framework for Touch Detection and Audio Feedback. Proc. ACM Hum.-Comput. Interact 5 , (2021), 33. DOI:https://doi.org/10.1145/3488550

[84] Matthieu Tixier, Charles Lenay, Gabrielle Le Bihan, Olivier Gapenne, and Dominique Aubert. 2013. Designing Interactive Content with Blind Users for a Perceptual Supplementation System. In Proceedings of the 7th International Conference on Tangible, Embedded and Embodied (TEI'13), February 10-13, 2013 , Barcelona, Spain. ACM Inc., New York, NY, 229-236. DOI:https://doi.org/https: //doi.org/10.1145/2460625.2460663

[85] Trint. Trint. Retrieved from https://rint.com/

[86] Marlon Twyman, Joe Mullenbach, Craig Shultz, J Edward Colgate, and Anne Marie Piper. 2015. Designing Wearable Haptic Information Displays for People with Vision Impairments. In Proceedings of the 9th International Conference on Tangible, Embedded, and Embodied Interaction (TEI'15), January 15-19, 2015 , Stanford, California. ACM Inc., New York, NY, 341-344. DOI:https://doi.org/https: //doi.org/10.1145/2677199.2680578

[87] Cathy Urquhart. 2017. Grounded Theory for Qualitative Research: A Practical Guide. DOI:https://doi.org/10.4135/9781526402196

[88] Jens Vetter. 2019. Tangible Signals - Physical Representation of Sound and Haptic Control Feedback. In Proceedings of the 13th International Conference on Tangible, Embedded, and Embodied Interaction (TEI'15), March 17-20, 2019, Tempe, Arizona. ACM Inc., New York, NY, 741-744. DOI:https://doi.org/10.1145/3294109. 3302958

[89] Jens Vetter. 2021. Tangible Signals - Prototyping Interactive Physical Sound Displays. In Proceedings of the 15th International Conference on Tangible, Embedded, and Embodied Interaction (TEI'21), February 14-17, 2021, Salzburg, Austria ACM Inc., New York, NY, 1-6. DOI:https://doi.org/10.1145/3430524.3442450

[90] James R. Wallace, Joseph Pape, Y.-L. Betty Change, Phillip J. McClelland, T.C. Nicholas Graham, Stacey D. Scott, and Mark Hancock. 2012. Exploring Automation in Digital Tabletop Board Games. In Proceedings of the ACM 2012 Conference on Computer Supported Cooperative Work Companion, February 11-15, 2012 Seattle, Washington. ACM Inc., New York, NY, 231-234. DOI:https://doi.org/https: //doi.org/10.1145/2141512.2141585

[91] Michele A Williams, Caroline Galbraith, Shaun K Kane, and Amy Hurst. 2014. "Just Let the Cane Hit It": How the Blind and Sighted See Navigation Differently. In Proceedings of the 16th International ACM SIGACCESS Conference on Computers \& Accessibility, October 20-22, 2014, Rochester, New York. ACM Inc., New York, NY, 217-224. DOI:https://doi.org/10.1145/2661334.2661380

[92] Nick Wingfield. 2014. High-Tech Push Has Board Games Rolling Again. The New York Times. Retrieved from https://www.nytimes.com/2014/05/06/technology/ high-tech-push-has-board-games-rolling-again.html?_r=1

[93] Jacob O. Wobbrock, Shaun K. Kane, Krzysztof Z. Gajos, Susumu Harada, and Jon Froehlich. 2011. Ability-based design: Concept, principles and examples. ACM Trans. Access. Comput. 3, 3 (April 2011). DOI:https://doi.org/10.1145/1952383. 1952384

[94] Qin Wu, Chenmei Yu, Yanjun Chen, Jiayu Yao, Xi Wu, Xiaolan Peng, and Teng Han. 2020. Squeeze the Ball: Designing an Interactive Playground towards Aiding Social Activities of Children with Low-Function Autism. In Proceedings of the 2020 CHI Conference on Human Factors in Computing Systems, April 25-30, 2020, Honolulu, HI. ACM Inc., New York, NY, 1-14. DOI:https://doi.org/10.1145/ 3313831.3376888

[95] Bei Yuan and Eelke Folmer. 2008. Blind Hero: Enabling Guitar Hero for the Visually Impaired. In Proceedings of the 10th International ACM SIGACCESS Conference on Computers and Accessibility, October 13-15, 2008, Halifax, Nova Scotia. ACM Inc., New York, NY, 169-176. DOI:https://doi.org/https://doi.org/10. 1145/1414471.1414503

[96] Bei Yuan, Eelke Folmer, and Frederick C Harris. 2011. Game Accessibility: A Survey. Univ. Access. Inf. Soc. 10, (June 2011), 81-100. DOI:https://doi.org/https: //doi.org/10.1007/s10209-010-0189-5

[97] 8 Different Types of Tabletop Games - Do You Know Them All? Board Game Friend. Retrieved October 12, 2021 from https://boardgamefriend.com/differenttypes-of-tabletop-games/

[98] 2009. The Braille Literacy Crisis in America Facing the Truth, Reversing the Trend, Empowering the Blind. 